\title{
Modeling the Radio Background from the First Black Holes at Cosmic Dawn: Implications for the $21 \mathrm{~cm}$ Absorption Amplitude
}

\author{
A. Ewall-Wice ${ }^{1,2}$ (i) , T.-C. Chang ${ }^{1,3}$, J. Lazio ${ }^{1}$, O. Doré ${ }^{1,3}$, M. Seiffert ${ }^{1}$, and R. A. Monsalve ${ }^{4,5,6,7,8}$ (i] \\ ${ }^{1}$ Jet Propulsion Laboratory, California Institute of Technology 4800 Oak Grove Dr, M/S 169-237, Pasadena CA 91109, USA \\ ${ }^{2}$ Dunlap Institute for Astronomy \& Astrophysics, 50 St. George St., Toronto, Ontario, M5S 3H4, Canada \\ ${ }^{3}$ California Institute of Technology, 1200 E California Blvd, Pasadena, CA 91125, USA \\ ${ }^{4}$ Department of Physics, McGill University, Montréal, QC H3A 2T8, Canada \\ ${ }^{5}$ McGill Space Institute, 3550 Rue University, Montréal, QC H3A 2A7, Canada \\ ${ }^{6}$ Center for Astrophysics and Space Astronomy, University of Colorado, Boulder, CO 80309, USA \\ ${ }^{7}$ School of Earth and Space Exploration, Arizona State University, Tempe, AZ 85287, USA \\ ${ }^{8}$ Facultad de Ingeniería, Universidad Católica de la Santísima Concepción, Alonso de Ribera 2850, Concepción, Chile \\ Received 2018 March 12; revised 2018 September 18; accepted 2018 September 26; published 2018 November 20
}

\begin{abstract}
We estimate the $21 \mathrm{~cm}$ radio background from accretion onto the first intermediate-mass black holes between $z \approx 30$ and $z \approx 16$. Combining potentially optimistic, but plausible, scenarios for black hole formation and growth with empirical correlations between luminosity and radio emission observed in low-redshift active galactic nuclei, we find that a model of black holes forming in molecular cooling halos is able to produce a $21 \mathrm{~cm}$ background that exceeds the cosmic microwave background $(\mathrm{CMB})$ at $z \approx 17$, though models involving larger halo masses are not entirely excluded. Such a background could explain the surprisingly large amplitude of the $21 \mathrm{~cm}$ absorption feature recently reported by the EDGES collaboration. Such black holes would also produce significant X-ray emission and contribute to the $0.5-2 \mathrm{keV}$ soft X-ray background at the level of $\approx 10^{-13}-10^{-12} \mathrm{erg} \mathrm{s}^{-1} \mathrm{~cm}^{-2} \mathrm{deg}^{-2}$, consistent with existing constraints. In order to avoid heating the intergalactic medium (IGM) over the EDGES trough, these black holes would need to be obscured by hydrogen column depths of $N_{\mathrm{H}} \sim 5 \times 10^{23} \mathrm{~cm}^{-2}$. Such black holes would avoid violating constraints on the CMB optical depth from Planck if their UV photon escape fractions were below $f_{\text {esc }} \lesssim 0.1$, which would be a natural result of $N_{\mathrm{H}} \sim 5 \times 10^{23} \mathrm{~cm}^{-2}$ being imposed by an unheated IGM.
\end{abstract}

Key words: dark ages, reionization, first stars

\section{Introduction}

The redshifted $21 \mathrm{~cm}$ line of neutral hydrogen (H I) offers a promising tool for mapping our universe's "cosmic dawn," when the first luminous sources formed (see Barkana \& Loeb 2001; Furlanetto et al. 2006; Morales \& Wyithe 2010; Pritchard \& Loeb 2012; McQuinn 2016 for reviews).

Several techniques are actively being pursued do detect the $21 \mathrm{~cm}$ signal. These include single dipole measurements of the sky-averaged "global signal," which is being carried out by experiments such as EDGES (Bowman \& Rogers 2010; Monsalve et al. 2017), SCI-H I (Voytek et al. 2014), BIGHORNS (Sokolowski et al. 2015), LEDA (Bernardi et al. 2016), and SARAS (Singh et al. 2017); and measurements of the power spectrum of temperature fluctuations using antenna arrays. Interferometric experiments seeking to measure the power spectrum include the GMRT (Paciga et al. 2013), the MWA (Dillon et al. 2014; Beardsley et al. 2016; Ewall-Wice et al. 2016; Jacobs et al. 2016; Trott et al. 2016), PAPER (Parsons et al. 2014; Ali et al. 2015; Jacobs et al. 2015), LOFAR (Patil et al. 2017), and HERA (DeBoer et al. 2017; Kohn et al. 2018). An alternative technique for accessing the $21 \mathrm{~cm}$ signal is to observe the absorption spectra of background radio sources (the " $21 \mathrm{~cm}$ Forest") by the intergalactic medium (IGM) (Carilli et al. 2002; Furlanetto \& Loeb 2002; Furlanetto 2006; Mack \& Wyithe 2012; Ciardi et al. 2013; Semelin 2016).

The EDGES collaboration has recently reported the detection of an absorption signature in the $21 \mathrm{~cm}$ global signal centered at redshift $z \approx 17$ (Bowman et al. 2018). The most striking feature of this detection might be its nominal depth of
$500 \mathrm{mK}$, roughly twice as deep as what has been predicted by previous canonical models (see Figure 1 of Cohen et al. (2017)). These models assume that the temperature of H I gas cannot cool below the adiabatic cooling limit for baryons decoupling from the cosmic microwave background (CMB) at redshift $z \approx 150$ (Peebles 1993) and that the CMB is the only significant $21 \mathrm{~cm}$ background at early times.

The dependence of the amplitude of the absorption signal on the radio background and gas temperature can be gleaned from the equation for the brightness temperature of $21 \mathrm{~cm}$ radio emission/absorption from a distant H I cloud (Madau et al. 1997),

$$
\delta T_{b} \propto\left(1-\frac{T_{\mathrm{CMB}}+T_{\mathrm{rad}}}{T_{s}}\right)
$$

where $T_{\mathrm{CMB}}$ and $T_{\mathrm{rad}}$ are the brightness temperatures of the $\mathrm{CMB}$ and any other $21 \mathrm{~cm}$ background at the redshift of the $\mathrm{H} \mathrm{I}$ cloud, and $T_{s}$ is the $21 \mathrm{~cm}$ spin temperature. The amplitude of the absorption signal can be increased by reducing $T_{s}$. In canonical models for the $21 \mathrm{~cm}$ signal, $T_{s}$ is primarily influenced by the Ly $\alpha$ and X-ray backgrounds. The former couples $T_{s}$ to the kinetic temperature and the latter heats the $\mathrm{H}$ I. Barkana (2018), Fialkov et al. (2018), and Muñoz \& Loeb (2018) recently explored the possibility that the large amplitude of the absorption signal might be explained by the kinetic temperature being lowered through baryonic dark matter interactions, first discussed by Tashiro et al. (2014) and Muñoz et al. (2015). Alternatively, the amplitude of the 
absorption feature could be boosted, above previous expectations, by an additional radio background.

During the completion of this manuscript, Feng \& Holder (2018) investigated the potential for $21 \mathrm{~cm}$ experiments to constrain the existence of a so far unexplained excess in the radio background measured by the ARCADE- 2 experiment (Fixsen et al. 2011). While the potential sources of this excess are varied, ranging from instrumental systematics to new astrophysics (see Singal et al. 2018a for an overview), Feng \& Holder (2018) calculated that even a small fraction of the reported excess originating beyond $z \gtrsim 17$ can cause a very significant increase in the $21 \mathrm{~cm}$ absorption feature amplitude. From Equation (1), we see that for fixed $T_{s}$, the presence of an additional backlight with temperature $T_{\text {rad }}$ will lead to a multiplicative increase in the absorption signal by a factor

$$
F_{\text {boost }} \approx 1+\frac{T_{\text {rad }}}{T_{\mathrm{CMB}}}
$$

when $T_{\mathrm{rad}}+T_{\mathrm{CMB}} \gg T_{s}$, where $T_{s}$ is the H I spin temperature. Thus, if a physical process can produce a radio background similar to or greater than the CMB, it can potentially explain the large absorption feature reported by Bowman et al. (2018). For instance, supernovae explosions of supermassive stars have been considered as a significant source of radio emission from $z \gtrsim 20$ (Biermann et al. 2014).

It has been suggested that the presence of radio emission from active galactic nuclei (AGNs) before or during the cosmic dawn might be detectable through its statistical imprint on the power spectrum from $21 \mathrm{~cm}$ forest absorption features (EwallWice et al. 2014) or in its direct impact on the $21 \mathrm{~cm}$ spin temperature (Bolgar et al. 2018). Both Ewall-Wice et al. (2014) and Bolgar et al. (2018) used models that extrapolated empirical trends in nearby radio sources (Haiman et al. 2004; Wilman et al. 2008) to predict the impact of these sources on the power spectrum but neither of these works addressed the global signal. Given the dramatic amplitude of the EDGES feature, it is clearly worth exploring whether it might be produced by radio emission from growing black hole seeds.

In this paper, we explore the potential contribution to a highredshift $(z \gtrsim 16)$ radio background that might exist from accreting black hole seeds during the cosmic dawn. We derive our results using a simple analytic framework that only considers growth through continuous accretion. This simplified view should be valid at early times given the relatively massive birth halos (virial temperatures of $T_{\text {vir }} \gtrsim 10^{3} \mathrm{~K}$ ) that we consider (Johnson et al. 2013). In Section 2, we present a simple motivational argument for the plausibility of black hole accretion producing the EDGES feature, before discussing our semi-analytic model for black hole seed formation and growth. In Section 3, we present calculations of our model's contribution to the cosmic X-ray background (CXB), faint radio source counts, and the $21 \mathrm{~cm}$ background experienced by $\mathrm{H}$ I during the cosmic dawn. We also explore the ionization and Lyman-Werner (LW) backgrounds that would be generated by these sources. In Section 4 we discuss several of the issues that our model faces as an explanation for the reported EDGES signal, and conclude in Section 5. Throughout this work, we assume the cosmological parameters from Planck Collaboration et al. (2016).

\section{Modeling the Radio Emission from Early Black Hole Accretion}

\subsection{Overall Motivation}

We begin with a simple calculation with which we demonstrate the plausibility of explaining the EDGES feature by radio emission from accreting black holes. The empirically motivated analysis that follows stems from three somewhat optimistic assumptions: (1) a non-negligible fraction of the known black hole mass already exists at high redshift; (2) these black holes are undergoing Eddington to super-Eddington accretion; (3) these early black holes have radio-loudnesses similar to what is observed in AGN at $z \approx 0$.

The emissivity of radio emission at redshift $z$ can be modeled as proportional to the mass density of black holes, $\rho_{\mathrm{bh}}$,

$$
\epsilon_{\nu}(z) \propto f_{\text {duty }}(z) f_{\text {edd }}(z) \rho_{\text {bh }}(z),
$$

where $f_{\text {edd }}(z)$ is the typical Eddington luminosity of black holes at redshift $z$, and $f_{\text {duty }}$ is the duty cycle. While black holes will be at significantly lower densities at high redshifts, their Eddington ratios and duty cycles may be significantly larger than the values found at $z \lesssim 1$. For example, Shen \& Kelly (2012) find that the typical Eddington ratios of broad line quasars increase from $\sim 10^{-2}$ at $z \approx 0$ to $f_{\text {edd }} \sim 0.3$ at $z \approx 4$. Meanwhile the duty cycle of black holes at $z \approx 0$ is $f_{\text {duty }} \sim 10^{-3}-10^{-2}$ (Shankar et al. 2009), while some models of black hole accretion in the early universe find consistent $f_{\text {duty }} \sim 1$ for $\sim 10^{8}$ yr (Pacucci et al. 2015). For the sake of argument, we assume that a significant fraction (1\%) of the black hole mass has already been assembled between $z \approx 25$ and $z \approx 17$. We assume that each black hole emits in X-rays at some fraction, $f_{\mathrm{X}}$, of the Eddington limit of $L_{\mathrm{E}}=1.26 \times$ $10^{31} \mathrm{~W} \mathrm{~Hz}^{-1}\left(M_{\mathrm{bh}} / M_{\odot}\right)$. If $L_{\mathrm{x}} \equiv f_{X} L_{\mathrm{E}}=0.1 L_{\mathrm{E}}$ is emitted in $\mathrm{X}$-rays for each radio-loud black hole between 0.1 and $2.4 \mathrm{keV}$, we can assign a radio luminosity according to the radio-quiet Fundamental plane in Wang et al. (2006), and boost the luminosity of radio-loud quasars $\left(f_{L}=10 \%\right.$ of the total population) by a factor of $10^{R}=10^{3}$ according to the typical radio-loudness found in SDSS/FIRST AGN by Ivezić et al. (2002). If $1 \%$ of the present-day black hole mass $\left(\sim 10^{4} h^{2} M_{\odot} \mathrm{Mpc}^{-3}\right)$ was accreting at high redshift, we obtain an emissivity,

$$
\begin{aligned}
& \epsilon(\nu, z) \approx 1.2 \times 10^{22}\left(\frac{f_{L}}{0.1}\right)\left(\frac{f_{\text {duty }}}{1}\right)\left(\frac{10^{R}}{10^{3}}\right)\left(\frac{f_{X}}{0.1}\right)^{0.86} \\
& \quad \times\left(\frac{\rho_{\text {bh }}}{10^{4} h^{2} M_{\odot} \mathrm{Mpc}^{-3}}\right)\left(\frac{\nu}{1.4 \mathrm{GHz}}\right)^{-0.6} \mathrm{~W} \mathrm{~Hz}{ }^{-1} h^{3} \mathrm{Mpc}^{-3} .
\end{aligned}
$$

An $\mathrm{H}$ I cloud at redshift $z$ would experience a specific intensity of

$$
J_{\nu}(z)=\frac{c(1+z)^{3}}{4 \pi} \int_{z}^{\infty} \epsilon\left[\nu \frac{1+z^{\prime}}{1+z}, z^{\prime}\right] \frac{d z^{\prime}}{\left(1+z^{\prime}\right) H\left(z^{\prime}\right)},
$$

where $H(z)$ is the Hubble parameter. Placing these sources between the redshifts $z=17$ and $z=25$, we compute the brightness temperature at redshift $z$ and $\nu=1420.41 \mathrm{GHz}$, 
$T_{\nu=1420.4 \mathrm{GHz}} \equiv T_{\mathrm{rad}}(z)$, using

$$
T_{\nu}(z)=\frac{c^{2} J_{\nu}(z)}{2 \nu^{2} k_{B}}
$$

and find that $T_{\text {rad }} \approx 90 \mathrm{~K}$, at $z=17$, nearly twice the CMB temperature at $z=17$. Thus, with $1 \%$ of the present-day black hole mass, we obtain a boost factor $F_{\text {boost }} \approx 3$, which is enough to explain the amplitude of the EDGES feature while leaving some room to spare for ionization and heating! We now proceed with a more physically motivated calculation of $\rho_{\mathrm{bh}}$ during the cosmic dawn, where we consider a broad range of black hole seeding, growth, and emission scenarios.

\subsection{Seeding Prescription}

How black holes quickly grew into the $10^{8}-10^{9} M_{\odot}$ supermassive black holes (SMBHs) observed only $\sim 1$ billion yr after the Big Bang remains a theoretical puzzle (Fan et al. 2003; Mortlock et al. 2011; Wu et al. 2015; Bañados et al. 2018). Explanations for the progenitors of these SMBHs generally follow two paradigms.

In the first paradigm, black hole seeds with masses of $\sim 10-10^{3} M_{\odot}$ are formed as the remnants of the first generation of Population III stars (Haiman \& Loeb 2001; Madau \& Rees 2001). Such Pop-III stars are expected to form with masses of $\sim 10-10^{3} M_{\odot}$ (Abel et al. 2002; Bromm et al. 2002; Yoshida et al. 2008; Stacy et al. 2010; Greif et al. 2011a; Hirano et al. 2015) in molecular cooling halos with $T_{\text {vir }} \lesssim 10^{4} \mathrm{~K}$ (Haiman et al. 1996; Abel et al. 1997; Tegmark et al. 1997). In order for these black holes to reach SMBH masses by $z \sim 7$, the accretion must proceed very efficiently, i.e., at or above the Eddington limit (Volonteri \& Rees 2005, 2006; Rhook \& Haehnelt 2006; Alexander \& Natarajan 2014; Madau et al. 2014; Volonteri et al. 2015).

In the second paradigm, black holes form with initial masses of $\sim 10^{4}-10^{5} M_{\odot}$. These seeds can arise from "direct collapse black holes" (DCBHs; Bromm \& Loeb 2003; Shang et al. 2010; Johnson et al. 2012), self-gravitating pre-galactic disks (Begelman et al. 2006; Lodato \& Natarajan 2006), or runaway stellar mergers (Devecchi \& Volonteri 2009; Davies et al. 2011; Stone et al. 2017).

We capture the order-of-magnitude characteristics of seeding through these scenarios using the following semi-analytic model. We model the evolution of a population of black hole seeds by constructing a grid of values of the Tinker et al. (2008) halo-mass function between an initial redshift at which the seed halo density is nearly zero (we choose $z_{\max }=80$ ) and $z_{\min }=16$. At each time step, we assume that black hole seeds can form in some fraction, $f_{\text {halo, of halos with masses }}$ (or virial temperatures) between some minimum $\mathbf{M}_{\min }$ and maximum $\mathbf{M}_{\max }$ values. We assign each formation halo a black hole seed with mass $\mathbf{M}_{\mathrm{bh}}=f_{\text {seed }} \mathbf{M}_{\text {halo. Iterating forward in }}$ time, we estimate the number of new seeds added to the total black hole population at each time step to be $\Delta n_{\text {seed }}=\dot{n}_{\text {seed }} \Delta t$, where

$$
\begin{aligned}
& \dot{n}_{\text {seed }}\left(\mathrm{M}_{\mathrm{bh}, \text { seed }}\right) \\
& \quad= \begin{cases}f_{\text {halo }} \dot{n}_{\text {halo }}\left(\frac{\mathrm{M}_{\text {bh,seed }}}{f_{\text {seed }}}\right) & \mathrm{M}_{\text {halo }} \in\left[\mathrm{M}_{\text {min }}, \mathrm{M}_{\text {max }}\right], \\
0 & \text { otherwise }\end{cases}
\end{aligned}
$$

Table 1

Black Hold Seeding Model Parameters

\begin{tabular}{lcccc}
\hline \hline Model & $f_{\text {seed }}$ & $f_{\text {halo }}$ & $M_{\min }$ & $M_{\max }$ \\
\hline Pop-III & $1.5 \times 10^{-4}$ & 0.1 & $2.1 \times 10^{3} \mathrm{~K}$ & $10^{4} \mathrm{~K}$ \\
DCBH & $10^{-2}$ & $10^{-4}$ & $10^{7} M_{\odot}$ & $10^{8} M_{\odot}$ \\
Unstable Clusters & $10^{-3}$ & $5 \times 10^{-2}$ & $10^{4} \mathrm{~K}$ & $10^{5} \mathrm{~K}$ \\
\hline
\end{tabular}

where $\dot{n}_{\text {halo }}$ is the number of halos that were below $\mathrm{M}_{\min }$ at $t-\Delta t$ and have accreted enough mass by time $t$ such that $M_{\text {halo }}=M_{\text {bh,seed }} / f_{\text {seed }} \geqslant M_{\text {min }}$. We compute the accretion rate of halos at each time step using the fitting formula of Fakhouri et al. (2010),

$$
\dot{M}_{\text {halo }}=46.1 M_{\odot} \mathrm{yr}^{-1}\left(\frac{M_{\text {halo }}}{10^{12} M_{\odot}}\right)^{1.1}(1+1.11 z) H(z) / H(0) \text {. }
$$

Table 1 summarizes the parameters for three seeding models, which we now describe in more detail.

\subsubsection{Pop-III Remnants}

We take Pop-III black hole seeds to form in halos having temperatures between $T_{\text {vir }}=2000 \mathrm{~K}$ and $T_{\text {vir }}=10^{4} \mathrm{~K}$. In principle, halos with masses down to several hundred $\mathrm{K}$ are capable of cooling through $\mathrm{H}_{2}$ and forming stars. However, the presence of baryonic dark matter velocity offsets can increase this mass threshold by a factor of as much as $\approx 3$ (Greif et al. 2011c; Stacy et al. 2011; Fialkov et al. 2012). We opt for a more conservative value of $\approx 2000 \mathrm{~K}$ also used by Tanaka et al. (2016). Our high-mass cutoff was chosen considering that the majority of atomic cooling halos are likely to have been formed from sub-halos that have already been metal-enriched (Johnson et al. 2008).

Tanaka et al. (2016) consider models in which Pop-III seeds arise in $1 \%$ to $100 \%$ of dark matter halos. We choose an intermediate value for the halo fraction, $f_{\text {halo }} \approx 0.1$.

What are our expectations for $f_{\text {seed }}$ ? The fraction of baryons that end up in stars within mini-halos is expected to be relatively low, and on average $f_{\star} \lesssim 10^{-3}$ (Haiman \& Bryan 2006; Choudhury \& Ferrara 2006; Visbal et al. 2015). Meanwhile, stars with initial masses above $\gtrsim 240 M_{\odot}$ are expected to directly collapse into high-mass black holes (Yoon et al. 2012). The fraction of stellar mass that becomes locked into such objects depends primarily on the Pop-III initial mass function. Many studies find that the first protostellar disks fragment into small clumps (Stacy et al. 2010; Clark et al. 2011; Greif et al. 2011b, 2012), but it is still unclear whether these small fragments typically migrated to the center of the disk, forming a 100-1000 $M_{\odot}$ star, or remained apart, forming much less massive objects. Hirano et al. (2015) find that at $z \gtrsim 18$, before the EDGES feature, between $70 \%$ and $100 \%$ of stars form above the direct collapse threshold (their Table 2) and while their 2D simulations could not properly account for disk fragmentation, Hosokawa et al. (2016) argued that fragmentation might actually increase final stellar masses by suppressing UV feedback through episodic accretion. We adopt the more conservative fraction of stellar matter that ends up in DCBHs of $10 \%$. Assuming $f_{\text {halo }}=0.1$, and $f_{\star}=10^{-3}$ across all halos with and without Pop-III formation yields $f_{\text {seed }} \Omega_{m} / \Omega_{b}=10^{-3}$. 
Multiplying by the baryon-matter density ratio gives us $f_{\text {seed }}=$ $1.5 \times 10^{-4}$.

The assumption of $10 \%$ high-mass black hole formation might be relaxed by reducing the minimal virial temperature of halos. Lowering $T_{\mathrm{vir}}^{\mathrm{min}}$ to $500 \mathrm{~K}$ yields a potential seed host density at $z=25$ that is $\approx 31$ times greater than if the minimum mass is $2000 \mathrm{~K}$, so if we adopted this lower virial temperature and assumed only $0.3 \%$ of Pop-III stars ended their lives by collapsing into high mass black holes, we would obtain similar black hole densities.

\subsection{2. $\mathrm{DCBHs}$}

We seed halos with masses between $10^{7}$ and $10^{8} M_{\odot}$ and assign a mass fraction of $f_{\text {seed }}=10^{-2}$, approximately reproducing the initial mass function of Ferrara et al. (2014) with seeds ranging from $10^{5}$ to $10^{6} M_{\odot}$. The abundance of formation sites for DCBHs is currently uncertain, typically thought to require an unpolluted halo subject to a LW background. Dijkstra et al. (2014) predicted an occurrence rate of $\sim 10^{-7}-10^{-5} h^{3} \mathrm{Mpc}^{-3}$ at $z=10$, while Agarwal et al. (2012) predicted significantly higher values of $\sim 10^{-3} h^{3} \mathrm{Mpc}^{-3}$. We use the fraction from Tanaka et al. (2016) and set $f_{\text {halo }} \approx 10^{-4}$, which yields a formation site density of $\sim 10^{-3} h^{3} \mathrm{Mpc}^{-3}$ at $z=10$, similar to the Agarwal et al. (2012)s scenario. It is possible that baryondark-matter velocity offsets might amplify the abundance of pristine atomic cooling halos and direct collapse holes (Hirano et al. 2017), and that such objects may also be able to form in metal-polluted halos (Dunn et al. 2018). Hence, the reader should consider our specific DCBH model as simply illustrative.

\subsubsection{Unstable Clusters}

We include this model to represent the scenario presented by Devecchi \& Volonteri (2009) in which mildly polluted atomic cooling halos, subject to a UV background, form dense clusters of stars in their cores. Runaway collisions in these clusters lead to the formation of $\sim 10^{3} M_{\odot}$ black hole seeds. Devecchi \& Volonteri (2009) found that these seeds form in $f_{\text {halo }} \approx 5 \times 10^{-2}$ of halos above $T_{\text {vir }}=10^{4} \mathrm{~K}$. To obtain a typical black hole mass of $1000 M_{\odot}$, we set the mass fraction to be $10^{-4}$ of the host halo mass.

\subsection{Growth through Accretion}

After forming, we allow each seed to grow through accretion, radiating at $L=\frac{\eta}{1-\eta} \dot{\mathrm{M}}_{\mathrm{bh}} c^{2}$ at some fraction of the Eddington rate, $L=f_{\text {edd }} L_{E}\left(\mathrm{M}_{\mathrm{bh}}\right)$, where $L_{E}=1.26 \times 10^{31} \mathrm{~W}\left(M_{\mathrm{bh}} / M_{\odot}\right)$, and $\eta$ is the radiative efficiency of accretion (the fraction of the infalling rest-energy that is radiated away). If the black hole accretes some $f_{\text {duty }}$ fraction of the time, the mass varies as (Johnson et al. 2013)

$$
\mathbf{M}_{\mathrm{bh}}(t)=\mathbf{M}_{\mathrm{bh}, \text { seed }} \exp \left(f_{\text {duty }} f_{\text {edd }} \frac{(1-\eta)}{\eta} \frac{t-t_{\text {seed }}}{\tau_{E}}\right),
$$

where $\tau_{E} \approx 0.45 \mathrm{Gyr}$ is the Eddington timescale and $t_{\text {seed }}$ is the time at which the seed formed. In order to keep our analysis simple, we assume that each seed grows continuously from accretion, which is a reasonable assumption for our model with seed halos of $T_{\text {vir }}>10^{3} \mathrm{~K}$ (Johnson et al. 2013). We consider the range of radiative efficiencies discussed in Milosavljević et al.
(2009), of 0.025 to 0.4 . Fixing the level of radio-loudness to what is observed locally, we find that only efficiencies $\eta \lesssim 0.1$ are capable of producing sufficient levels of radio emission. We restrict our analysis to $0.03 \leqslant \eta \leqslant 0.05$ in the following discussion (we will briefly revisit large $\eta$ solutions in Section 4). We also consider a range of $f_{\text {edd }}$ between $10^{-2}$ and 1 and fix $f_{\text {duty }}=0.5$ which is an optimistic but reasonable assumption at high redshifts (Shankar et al. 2010).

\subsection{Radio Emission}

We assume that each black hole has an intrinsic X-ray luminosity between 2 and $10 \mathrm{keV}$ given by $L_{2-10}=k_{\text {bol }}^{-1} f_{\text {edd }} L_{\mathrm{E}}$ between 2 and $10 \mathrm{keV}$, where we use a bolometric correction factor of $k_{\mathrm{bol}}^{-1}=0.07$, consistent with the distribution observed by Lusso et al. (2010). We then extrapolate to $L_{X} \equiv L_{0.1-2.4}$ by assuming that the X-ray luminosity follows a power law with index of 0.9 .

The nature of radio emission from accreting black holes is still poorly understood. Thus, we will employ empirical trends. The radio luminosity (associated with jet emission) of an accreting black hole, relative to its optical luminosity (associated with the accretion disk), is typically described by a radio-loudness parameter, $R$, which either refers to the logarithm of the ratio between $5 \mathrm{GHz}$ and $\mathrm{B}$ band $(4250 \AA$ ) luminosities (Kellerman et al. 1989) or $1.4 \mathrm{GHz}$ and i-band $(8000 \AA)$ ratios (Ivezić et al. 2002). We adopt the latter definition. There is a lively debate as to whether the $R$ distribution is bimodal, consisting of two physically distinct radio-quiet and radio-loud populations, where the latter sources are typically $\sim 1000$ times more luminous than radio-quiet sources (Ivezić et al. 2002, 2004; Cirasuolo et al. 2003; Singal et al. 2018b). There is also disagreement as to whether the radio-loudness distribution evolves over redshift and in which direction (Jiang et al. 2007; Donoso et al. 2009; Singal et al. 2018b; Bañados et al. 2015). Correlation between radio emission and X-ray emission is also well-documented, primarily in the "fundamental plane" of black holes (Merloni et al. 2003) which, in most works, does not distinguish between radio-quiet and radio-loud sources. Wang et al. (2006) found that radio-loud sources tend to lie far above the trends fitted with radio-quiet sources.

The task of this work is not to better understand our expectations for radio-loudness at high redshift but rather to investigate what plausible levels of radio-loudness can produce the EDGES feature. To this end, we adopt the bimodal radioloudness model of Ivezić et al. (2002) and divide our black hole population into $10 \%$ radio-loud, and $90 \%$ radio-quiet, assuming that the radio-loud distribution does not evolve with redshift. Given $L_{X}$, we assign each black hole a radio luminosity at $1.4 \mathrm{GHz}$ using the radio-quiet fundamental plane (Merloni et al. 2003; Falcke et al. 2004) fitted in Wang et al. (2006) for radio-quiet AGNs:

$$
\begin{aligned}
L_{1.4}\left(M_{\mathrm{bh}}\right)= & \frac{8.3 \times 10^{-6}}{1.4 \times 10^{9} \mathrm{~Hz}}\left(\frac{L_{X}}{L_{\mathrm{E}}\left(M_{\mathrm{bh}}\right)}\right)^{0.86} L_{\mathrm{E}}\left(M_{\mathrm{bh}}\right) \\
& \times \begin{cases}1 & \text { radio quiet } \\
\left\langle 10^{0.83 R}\right\rangle & \text { radio loud }\end{cases}
\end{aligned}
$$

where the multiplicative ratio for radio emission from radioloud and radio-quiet AGNs predicted by the fundamental plane 


\section{Pop-III Clusters DCBH}
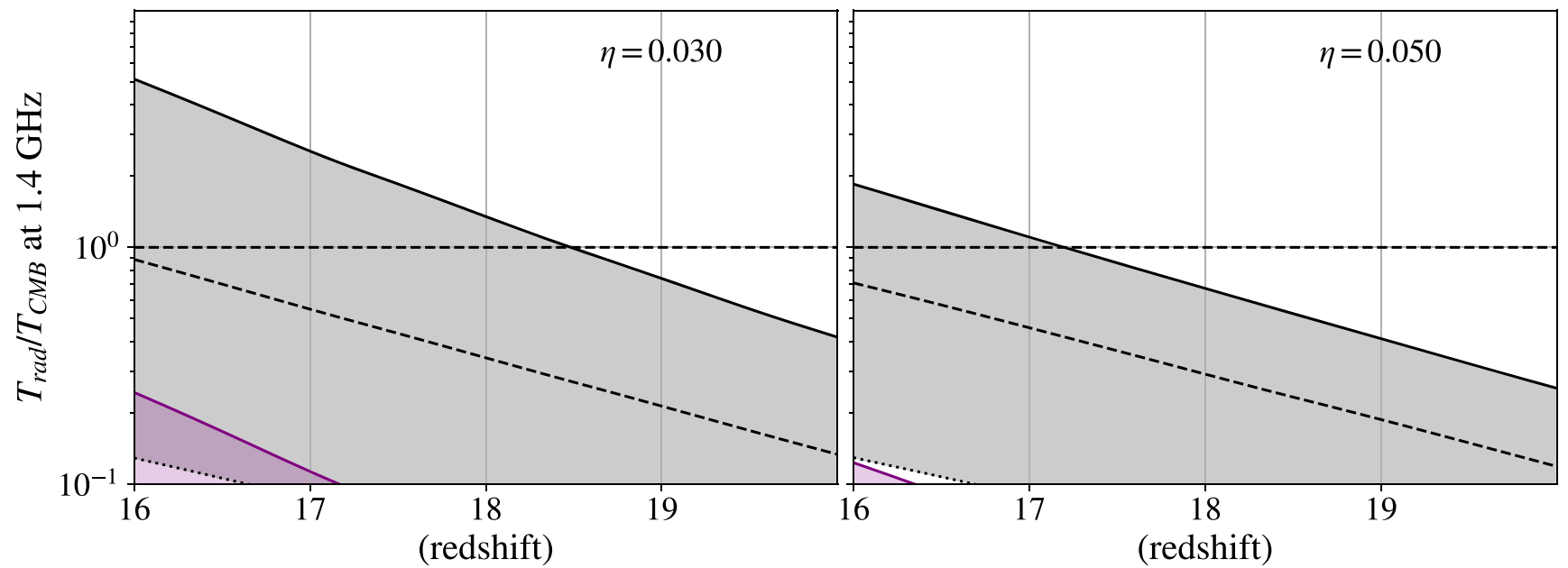

Figure 1. Ratio between $T_{\mathrm{rad}}$ and $T_{\mathrm{CMB}}$, as a function of redshift for $\eta=0.03$ (left) and $\eta=0.05$ (right). The filled shaded regions denote the range of temperatures predicted between $f_{\text {edd }}=10^{-1}$ and 1 for our Pop-III (gray), DCBH (red), and cluster-collapse (purple) scenarios (see Table 1). In the absence of heating, models that pass above the horizontal dashed line at $z \lesssim 17$ are producing a sufficient radio background to account for the absorption feature detected by the EDGES experiment.

scales as $\sim 10^{0.83 R}$. We calculate the average $\left\langle 10^{0.83 R}\right\rangle$ radio luminosity for our radio-loud population by modeling $e^{R}$ as $\log$-normal distributed with a mean of $\langle R\rangle=2.8 \times \ln (10)$ and a standard deviation of $\sigma=1.1 \times \ln (10)$, which describes the distribution of radio-loudness for SDSS/FIRST AGN at redshift $\approx 1$ (see Figure 19. of Ivezić et al. (2002)). This radio-loudness distribution yields a boost factor of $\left\langle 10^{0.83 R}\right\rangle \approx$ $1.9 \times 10^{3}$ for radio-loud AGNs.

To match the spectral index of the observed radio background, we assign our radio sources a spectral index of -0.6 . This value is within the range of what one would expect for synchrotron emitted by shock-injected electrons, such as what is observed in the lobes and hot-spots of low-redshift radio sources. However, diffuse lobe emission may be difficult to produce at high redshifts due to enhanced Compton cooling from CMB photons (Ghisellini et al. 2014, 2015; Saxena et al. 2017). Sharma (2018) notes that the short cooling times at high redshift would lead to spectral aging and an index closer to $\approx-1.1$. Our computed radio background amplitude at $z \approx 18$ is not very sensitive to the choice of spectral index, although we note that the higher value suggested by Sharma (2018) would help our scenario obey radio source count constraints by making the sources observed at $z=0$, and higher rest-frame frequency appear fainter.

\section{Results}

We now present our results. We start by presenting our model's contribution to the $21 \mathrm{~cm}$ radio background (Section 3.1). We then discuss the predictions our model makes for faint radio source counts (Section 3.2), the soft X-ray background (Section 3.3), the density of black holes between redshifts of 20 and 16 (Section 3.4), and our model's implications for reionization (Section 3.5) and LW feedback (Section 3.6).

\subsection{The Impact on Radio Background and $21 \mathrm{~cm}$ Signal During the Cosmic Dawn}

We compute the boost factor for the $21 \mathrm{~cm}$ absorption trough (assuming no heating from X-rays) given in Equation (2). This quantity provides us with a plausible upper limit on the amount by which our black holes can boost the $21 \mathrm{~cm}$ signal.

We plot $T_{\mathrm{rad}} / T_{\mathrm{CMB}}$, at $21 \mathrm{~cm}$, in Figure 1 for $\eta=0.03$ and $\eta=0.05$, with the filled regions denoting Eddington ratios between $f_{\text {edd }}=10^{-2}$ and 1 . It is apparent that among our black hole models, only our Pop-III scenario produces a radiative background close to the CMB and capable of producing the EDGES absorption feature. If a radiative background from accreting black holes is responsible for the observed $21 \mathrm{~cm}$ absorption amplitude of $\approx 500 \mathrm{mK}$, then it is unlikely that these objects were constrained to form in halos above the atomic cooling threshold, at least under the assumptions of our DCBH and cluster models.

We conclude, from Figure 1, that we can produce enough radio background to explain the EDGES feature while still satisfying the CXB constraints with Pop-III black holes that formed in $10 \%$ of eligible halos before and during the cosmic dawn. These black holes need to have accreted with a duty cycle of $f_{\text {duty }} \approx 0.5$ and radiative efficiency of $\eta \lesssim 0.05$, while radiating with an Eddington efficiency between $f_{\text {edd }} \approx 0.1$ and 1. These numbers can be relaxed if we allow for higher radio emission efficiencies or a larger radio-loud fraction.

From Figure 1, it is clear that DCBH and cluster-collapse black holes cannot produce enough radio emission to explain the EDGES absorption feature unless they formed with significantly higher efficiencies than is theoretically expected. We find that for $f_{L}=0.1$, cluster-collapse black holes approach necessary levels of radio emission if $f_{\text {halo }} \approx 0.5$, while DCBH's require $f_{\text {halo }} \approx 10^{-2}$.

It is worth briefly discussing the contribution of our model to the radio background observed at $z=0$. Our most emissive model, $\eta=0.03, f_{\text {edd }}=1$, produces a brightness temperature, at $1.4 \mathrm{GHz}$, of $\approx 8 T_{\mathrm{CMB}}$ at $z=16$, roughly $371 \mathrm{~K}$. At $z=0$, this would be observed as $21.84 \mathrm{~K}$ emission at $83 \mathrm{MHz}$. 


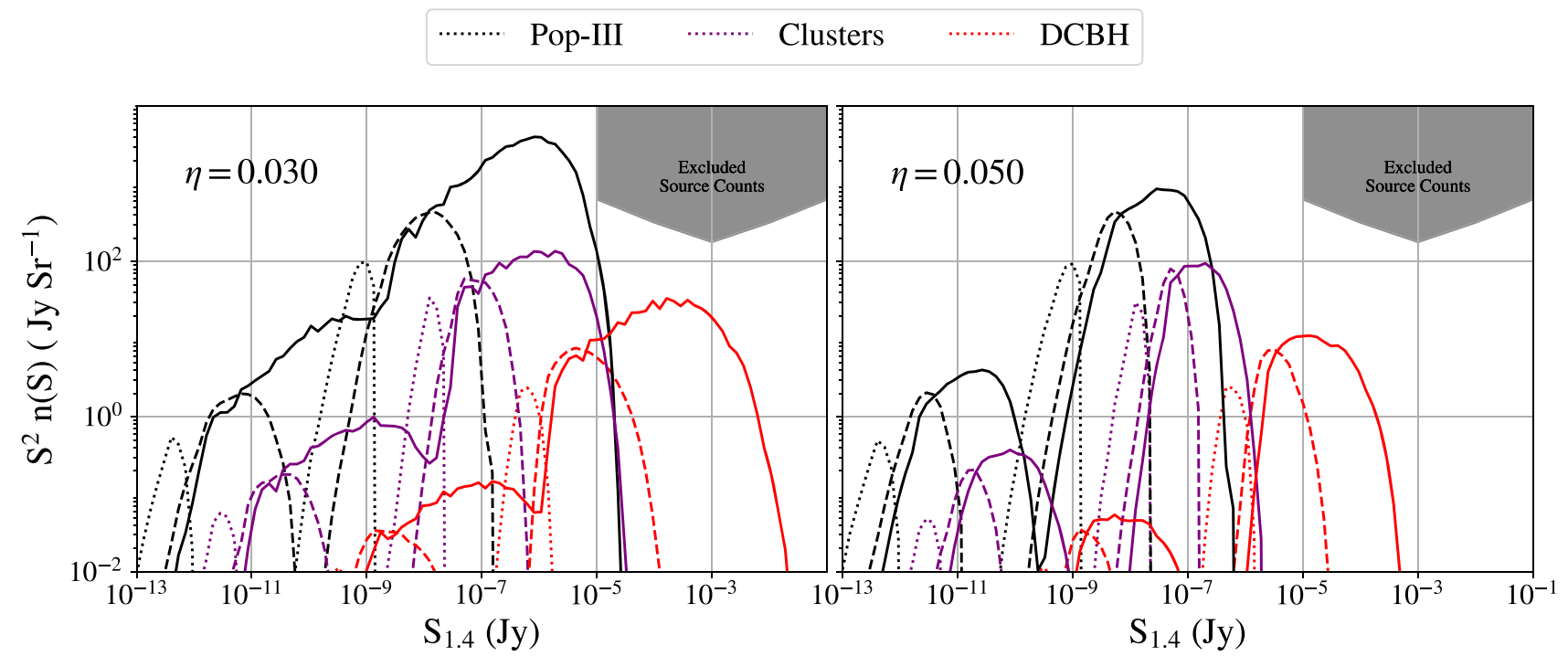

Figure 2. Number of sources per steradian per flux interval on the sky from $z \geqslant 16$, times flux-squared, for our various models of black hole seeding and growth. The solid lines indicate $f_{\text {edd }}=1$, while the dashed and dotted lines assume $f_{\text {edd }}=10^{-1}$ and $f_{\text {edd }}=10^{-2}$, respectively. Left column: $\eta=0.03$. Right column: $\eta=0.05$. The gray shaded region indicates flux counts excluded by Condon et al. (2012). All of our scenarios predict flux counts below existing limits. Reducing the radiative efficiency to $\approx 0.03$ leads to our $f_{\text {edd }} \approx 1$ scenario violating existing point-source constraints, though a steeper spectral index might still allow for it.

For our spectral index of -0.6 in flux density, this gives $T_{\text {rad }}(\nu, z=0) \approx 33(\nu / 1 \mathrm{GHz})^{-2.6} \mathrm{mK}$, over an order of magnitude below the claimed detection of an $\approx 1 \mathrm{~K}$ excess observed by ARCADE-2 (Fixsen et al. 2011). This result is consistent with Feng \& Holder's (2018) observation that background temperatures significantly below those measured by ARCADE-2 can explain a large increase in the absorption feature.

\subsection{Radio Source Counts}

In Figure 2 we show the number of sources per flux interval and solid angle on the sky observed at $z=0$ from our radio sources at $z \geqslant 16$ at $1.4 \mathrm{GHz}$. All of our models show two peaks in flux corresponding to the radio-loud and radio-quiet sub-populations. Low radiative efficiencies and high Eddington rates lead to larger populations of more massive black holes, forming high-flux wings. Our Pop-III models yield fluxes below the detection threshold of any existing surveys, $\lesssim 10^{-5} \mathrm{Jy}$, while our cluster and DCBH scenarios lead to $\sim \mathrm{mJy}$ sources that only contribute to the detected source counts at the $1 \%-10 \%$ level. All scenarios considered yield populations of sources below the limits on source counts imposed by surveys (e.g., Condon et al. 2012; Vernstrom et al. 2014).

\subsection{Soft X-Ray Background}

Our model also predicts the contribution from our accreting black holes to the CXB (McCammon et al. 2002; Hickox \& Markevitch 2006; Lehmer et al. 2012) (CXB). Fialkov et al. (2017) noted that when one subtracts the sources at $z \lesssim 10$ considered by Cappelluti et al. (2012), the X-ray flux from the cosmic dawn should not exceed $J_{0.5-2 \mathrm{keV}} \approx 2.5 \times$ $10^{-13} \mathrm{erg} \mathrm{s}^{-1} \mathrm{~cm}^{-2} \mathrm{keV}^{-1} \mathrm{deg}^{-2}$ over the $0.5-2 \mathrm{keV}$ band. We compute $\epsilon_{\nu}$ from our black hole population through

$$
\epsilon(\nu, z)=\int d M_{\mathrm{bh}} n\left(M_{\mathrm{bh}}\right) L_{\nu}\left(M_{\mathrm{bh}}\right)
$$

where $n\left(M_{\mathrm{bh}}\right)$ is the number of black holes of a given mass, and $L_{\nu}\left(M_{\mathrm{bh}}\right)$ is the luminosity of emission from the black hole at frequency $\nu$. We extrapolate the $0.1-2 \mathrm{keV}$ X-ray luminosities

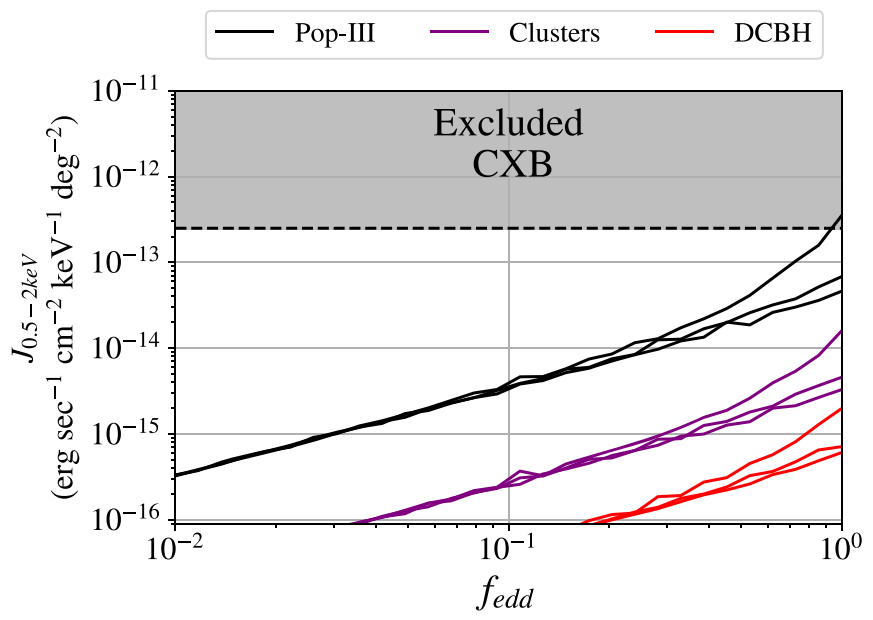

Figure 3. Integrated $\mathrm{X}$-ray background between 0.5 and $2 \mathrm{keV}$ predicted from our black hole models assuming obscuration below $0.5 \mathrm{keV}$ and a spectral index of 0.9 for an accreting black hole population above $z=16$. Each set of lines for each seeding model corresponds to radiative efficiencies of $0.4,0.05$, and 0.03 with background amplitudes increasing with decreasing radiative efficiency. All of our models fall below the X-ray background constraint from Fialkov et al. (2017; black dashed line).

from Wang et al. (2006) to harder energies (which are redshifted into the $0.5-2 \mathrm{keV}$ band by $z=0$ ) by assuming an X-ray spectral index of 0.9, typical of AGNs (Nandra \& Pounds 1994; Reeves \& Turner 2000; Page et al. 2005; Piconcelli et al. 2005), and a minimal X-ray energy of $0.5 \mathrm{keV}$ due to self-absorption by the interstellar medium in each black hole's host galaxy (Mesinger et al. 2013; Fialkov et al. 2014; Pacucci et al. 2014; Das et al. 2017). We report $J_{0.5-2 \mathrm{keV}}$ versus $f_{\text {edd }}$ in Figure 3 . We find that our models mostly lie below the $\mathrm{X}$-ray background constraint.

\subsection{Black Hole Densities}

We next inspect the density of black holes produced by our accretion/emission models in Figure 4. By redshift 16, the 


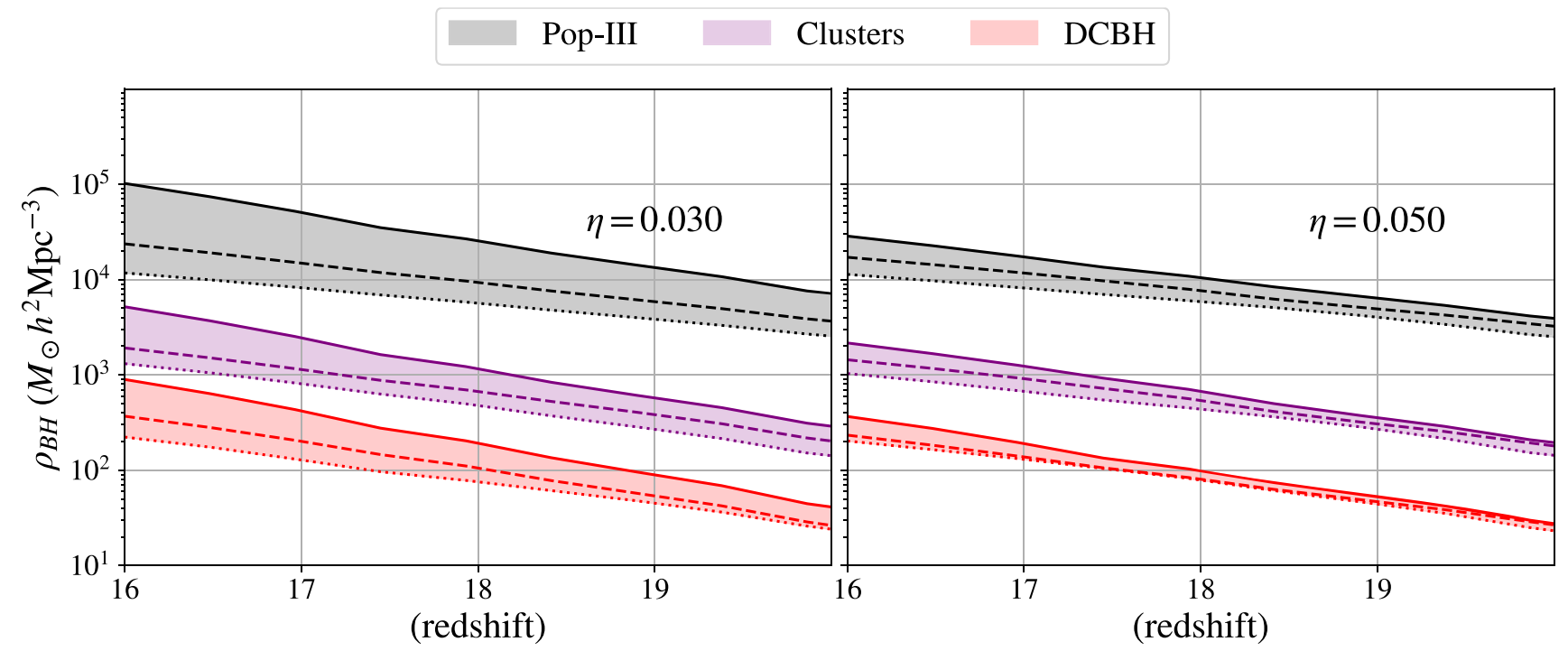

Figure 4. Comoving density of black holes produced by our formation model. In all cases, they are below the limit determined from dynamical black hole masses in the Local Universe $\left(\approx 1.1 \times 10^{6} \mathrm{M}_{\odot} h^{2} \mathrm{Mpc}^{-3}\right)$, though allowing for accretion to proceed unregulated would violate these constraints at lower redshifts. Shaded regions indicate densities obtained between the Eddington ratios of $10^{-2}$ and 1 for each set of models.

most optimistic Pop-III models, which might produce or overproduce the EDGES signature, are approaching the limit of $\approx 1.1 \times 10^{6} M_{\odot} h^{2} \mathrm{Mpc}^{-3}$ determined from dynamical black hole masses in the Local Universe (Merritt \& Ferrarese 2001). It follows that if these accreting sources are responsible for the EDGES amplitude, then their growth and emission must be curtailed through some feedback process at lower redshifts.

\subsection{Contribution to Reionization}

Seeing that it is possible for our Pop-III model to produce a sufficient radio background to explain the amplitude of the EDGES absorption feature, we investigate this model's implications on reionization. From each quasar's X-ray luminosity at $2 \mathrm{keV}$, we assign an optical luminosity at $2500 \AA$ using the relationship observed by Lusso et al. (2010),

$$
\log _{10} L_{2500 \AA}=\log _{10} L_{2 \mathrm{keV}}+2.605 \times \alpha_{o x},
$$

where we use their empirically derived value of $\alpha_{\text {ox }}=1.37$. We compute the ionizing flux by extrapolating from $2500 \AA$ to $912 \AA$ using a power law of -0.65 and assume a steeper spectrum blueward of $912 \AA$ with a spectral index of -1.7 (Lusso et al. 2015). We compute the rate of hydrogen-ionizing photons as $\dot{n}_{\text {ion }}(z)=f_{\text {esc }} \int \frac{d \nu}{h_{P} \nu} \epsilon[\nu, z]$, where $h_{P}$ is Planck's constant and $f_{\text {esc }}$ is the fraction of ionizing photons that are able to escape into the IGM.

The escape fraction of high-redshift galaxies remains highly uncertain. Ma et al. (2015) found escape fractions between 0.01 and 0.05 for $\sim 10^{9} M_{\odot}$ halos in simulations, while observations of larger galaxies at lower redshifts found escape fractions $f_{\text {esc }} \sim 10^{-2}-0.5$ (Bridge et al. 2010; Izotov et al. 2016; Vanzella et al. 2016; Vasei et al. 2016; Grazian et al. 2017). These may not be representative of our Pop-III hosts, which have masses of $\approx 10^{6} M_{\odot}$ when our seeds are formed, and grow to between $\approx 10^{8}$ and $\approx 10^{9} M_{\odot}$ by redshift 16 . Theoretical models of quasar-driven reionization often assume that the escape fraction of galaxies hosting quasars was significantly higher than their non-quasar hosting counterparts and take $f_{\text {esc }} \sim 1$ (e.g., Madau \& Shull (1996), Madau \& Haardt (2015)). Despite this, recent observations by Micheva et al. (2017) found $f_{\mathrm{esc}} \lesssim 10^{-2}$ for $\mathrm{AGN}$ at $z \approx 3$.

In order to prevent X-rays from escaping and heating the IGM, any black holes producing the EDGES feature would need to have $f_{\mathrm{esc}} \sim 0$ (see the discussion in Section 4). However, it is also possible that the black holes were not as obscured as our heating constraint suggests, but were extraordinarily radio-loud instead, overcoming any simultaneous heating. Thus, to be conservative, we consider escape fractions between 0.05 and 0.2 , much larger than the value imposed by our heating constraint but more in line with observations of lower-redshift sources.

Following Madau \& Haardt (2015), we assume that photons between 1 and 4 Ryd contribute primarily to hydrogen ionization, while photons above 4 Ryd contribute to helium ionization. We then obtain the volumetric filling fractions of $\mathrm{H}_{\mathrm{II}}$ $\left(\mathrm{Q}_{\mathrm{H} \mathrm{II}}\right)$, and $\mathrm{He}_{\mathrm{III}}\left(\mathrm{Q}_{\mathrm{He} \mathrm{III}}\right)$ by integrating the ionization equations (see Madau \& Haardt (2015) and references therein),

$$
\begin{gathered}
\dot{\mathrm{Q}}_{\mathrm{H} \mathrm{II}}=\frac{\dot{n}_{\mathrm{ion}}}{\left\langle n_{\mathrm{H}}\right\rangle}-\frac{\mathrm{Q}_{\mathrm{H} \mathrm{II}}}{t_{\mathrm{rec}, \mathrm{H}}} \\
\dot{\mathrm{Q}}_{\mathrm{He} \text { III }}=\frac{\dot{n}_{\text {ion }}}{\left\langle n_{\mathrm{He}}\right\rangle}-\frac{\mathrm{Q}_{\mathrm{He} \mathrm{III}}}{t_{\mathrm{rec}, \mathrm{He}}},
\end{gathered}
$$

where $\left\langle n_{\mathrm{H}}\right\rangle$ is the comoving number density of hydrogen atoms and $\left\langle n_{\mathrm{He}}\right\rangle$ is the comoving number density of helium. We use these authors' expressions for the recombination times of hydrogen, $t_{\mathrm{rec}, \mathrm{H}}$, and helium, $t_{\mathrm{rec}, \mathrm{He}}$, and halt the evolution of $\mathrm{Q}_{\mathrm{H} \text { II } / \mathrm{He} \text { III }}$ at $z$ such that $\mathrm{Q}_{\mathrm{H} \text { II } / \mathrm{He} \text { III }}=1$ or $z=16$, when we stop growing our black holes, whichever occurs first.

In Figure 5, we show the evolution of the neutral fraction, $x_{\mathrm{H}} 1-\mathrm{Q}_{\mathrm{H} \text { II }}$, with redshift for three different values of the escape fraction, $f_{\mathrm{esc}}=0.2, f_{\mathrm{esc}}=0.1$, and $f_{\mathrm{esc}}=0.05$.

For $f_{\text {esc }}=0.1$, all of our models introduce non-negligible amounts of ionization by $z=17$, with $x_{\mathrm{H} \text { I }}$ ranging from 0.4 , for our most emissive model, to $\approx 0.9$ at lower Eddington ratios. While this means that the overall amplitude of the absorption feature would fall by a factor of $\approx 2-4$, these very emissive models produce enough excess radio emission 


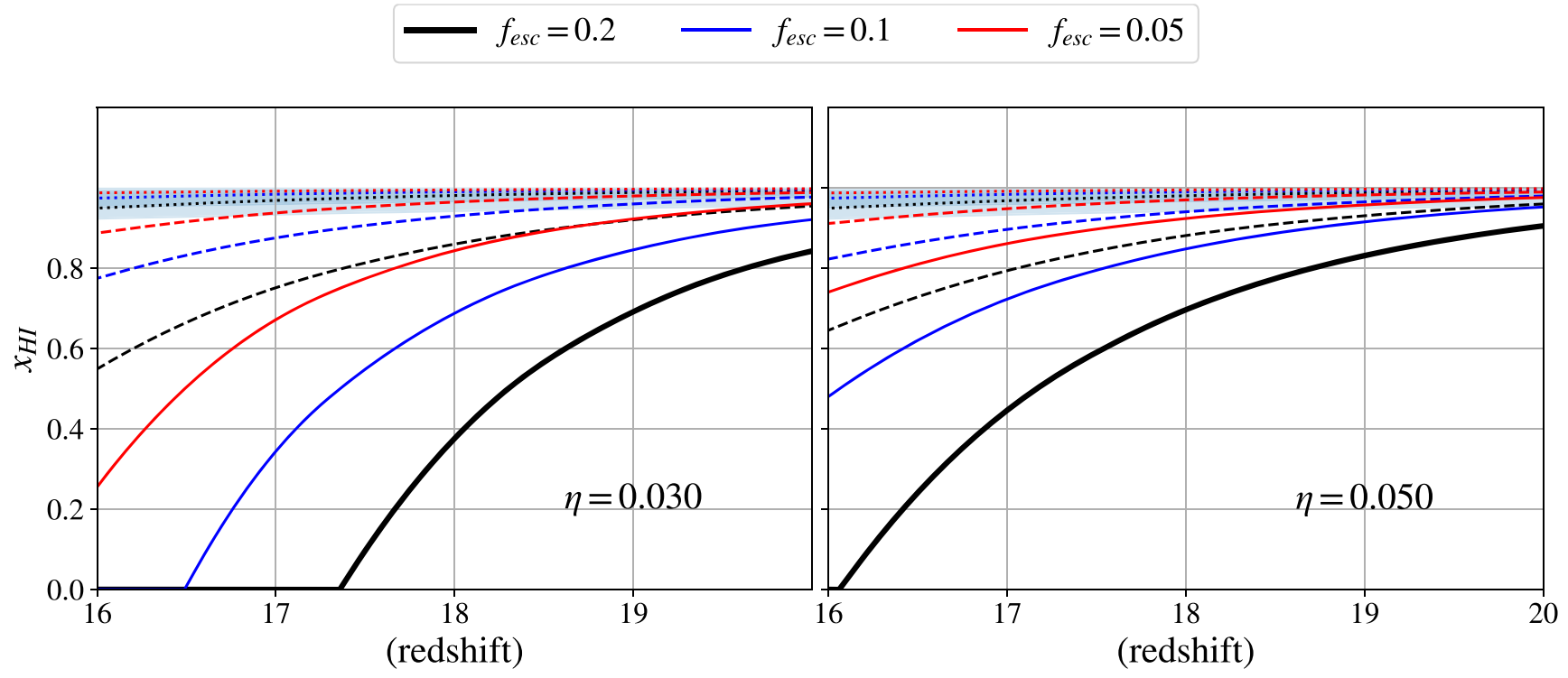

Figure 5. Evolution of the hydrogen neutral fraction, $x_{\mathrm{H}}$, for the ionizing flux of our Pop-III model and escape fractions of 0.2 (black lines), 0.1 (blue lines), and 0.05 (red lines). The dotted lines correspond to $f_{\text {edd }}=10^{-2}$, the dashed lines correspond to $f_{\text {edd }}=10^{-1}$, and the solid lines correspond to $f_{\text {edd }}=1$. The models that are able to produce the EDGES absorption feature also reduce the neutral fractions at $z=17$. In particular, for $\eta=0.03$ (left panel) and $f_{\text {edd }}=1$, the neutral fraction is $\approx 0.7$ for $f_{\text {esc }}=0.05$ and $\lesssim 0.4$ for $f_{\text {esc }}=0.1$. The blue shaded regions denote the $1 \sigma$ and $2 \sigma$ constraints on $x_{\mathrm{H} \mathrm{I}}$ from Millea \& Bouchet (2018). Demanding that the IGM remains unheated implies $f_{\text {esc }} \approx 0$, far lower than the models considered in this plot.

$$
-f_{\text {esc }}=0.2-f_{\text {esc }}=0.1-f_{\text {esc }}=0.05
$$
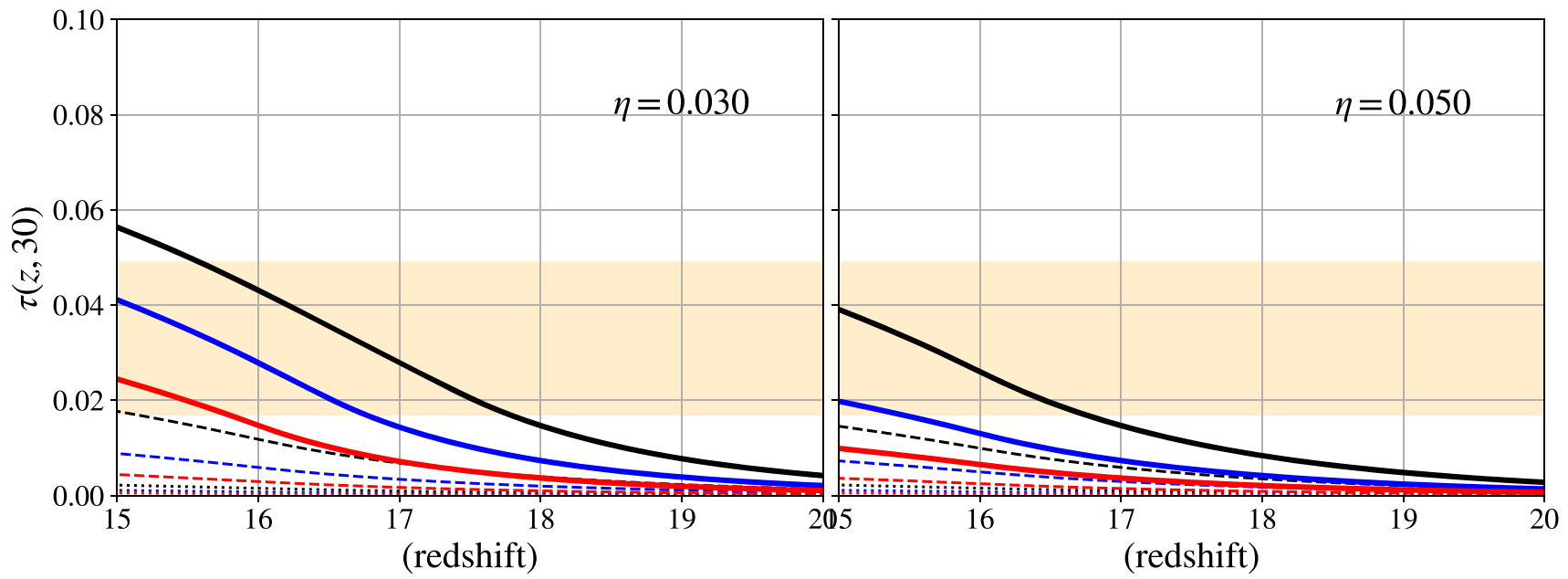

Figure 6. Integrated optical depth between $z$ and $z=30$ predicted by our Pop-III black hole model, assuming that accretion shuts down at $z=16$ and escape fractions $f_{\text {esc }}=0.05$ (red lines), $f_{\text {esc }}=0.1$ (blue lines) and $f_{\text {esc }}=0.2$ (black lines). The dotted lines correspond to $f_{\text {edd }}=10^{-2}$, the dashed lines correspond to $f_{\text {edd }}=10^{-1}$, and the solid lines correspond to $f_{\text {edd }}=1$. The orange shaded region denotes the range of $\tau(15,30)$ allowed by Heinrich $\&$ Hu (2018). The obscuration necessary for keeping the IGM unheated for the duration of the trough $(\sim 100 \mathrm{Myr})$ would naturally lead to $f_{\text {esc }}$ values much smaller than any considered in this plot.

(Figure 1) to make up for such a reduction. We also see that increasing $f_{\text {esc }}$ to $\approx 0.2$ can cause ionization to occur too early, erasing the absorption feature.

We conclude that with $f_{\text {esc }} \lesssim 0.1$, the ionizing flux is not problematic for the amplitude of the absorption feature. Furthermore, it is possible that fine-scale, optically thick substructures, such as Damped Ly $\alpha$ systems, can delay ionization further by $\Delta z \approx 2$ (Sobacchi \& Mesinger 2014).

While moderate ionization at high redshifts can still allow for the EDGES amplitude, it might come into conflict with the Thomson scattering optical depth of CMB photons measured by Planck Collaboration et al. (2016). Rather than calculate $\tau$ to $z=0$ from our black holes, which would only contribute to the part of the total ionization that is mainly expected from star formation at lower redshifts, we calculate the integrated optical depth between $z$ and $z_{\max }=30, \tau(z, 30)$, for which Planck polarization constraints of $\tau(15,30)=0.033 \pm 0.016$ have recently been derived (Heinrich \& Hu 2018). We show the results in Figure 6. Our $f_{\text {esc }} \approx 0.1$ models tend to be consistent with Heinrich \& Hu's (2018) results, though more recent work by Millea \& Bouchet (2018), whose $x_{\mathrm{H} \mathrm{I}}$ predictions we show in Figure 5, arrive at more stringent constraints, which would only allow for $f_{\text {esc }} \lesssim 0.05$ if accretion was Eddington-limited. 


\section{Pop-III $\square$ Clusters $\square$ DCBH}

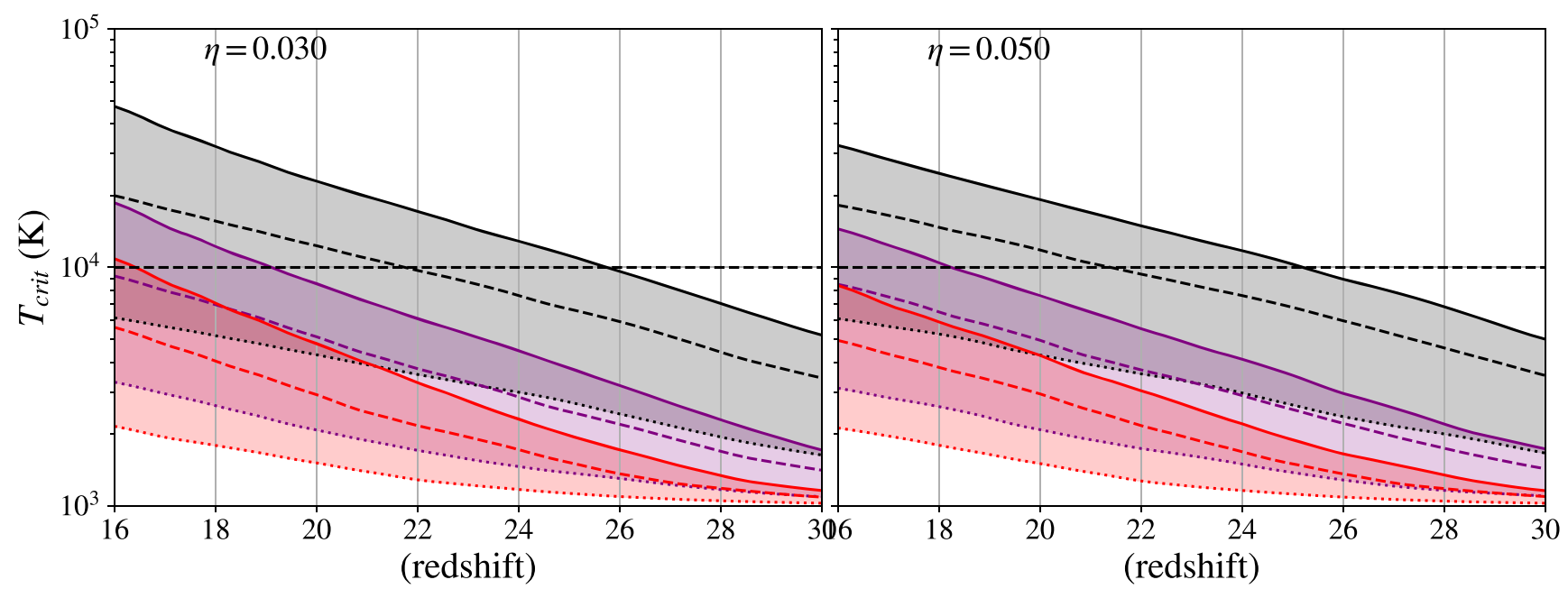

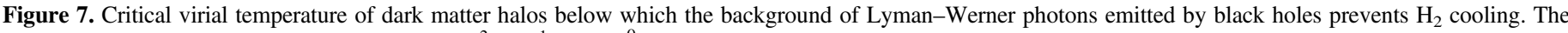

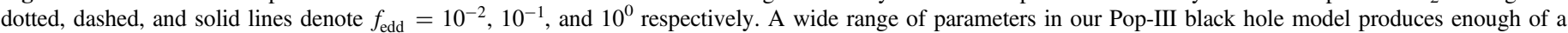

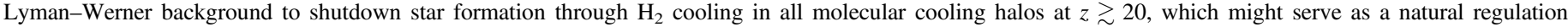
mechanism that prevents the overproduction of black holes.

\section{6. $L W$ Feedback}

Unlike their ionizing counterparts, LW photons between 11.2 and $13.6 \mathrm{eV}$ are capable of escaping into the IGM and dissociating the $\mathrm{H}_{2}$ molecules necessary for the cooling and collapse of baryons onto mini-halos (Haiman et al. 1997). Thus, a sufficiently strong LW background generated by early black holes can provide a natural feedback mechanism for scenarios where the black hole seeds form from Population-III stars.

We use the UV SED described in Section 3.5 to predict the LW background generated by the accreting black holes as a function of redshift using Equation (5), with the upper integration limit of $z_{m}$, where $z_{m}+1=1.04 \times(z+1)$, to account for the fact that LW photons can only redshift by a factor of $\approx 4 \%$ before being absorbed by a Lyman transition (Visbal et al. 2014). In Figure 7, we plot the virial temperature corresponding to the minimum halo mass that can provide enough self-shielding to allow for $\mathrm{H}_{2}$ cooling to occur within a Hubble time, $T_{\text {crit }}$, approximated by Equation (4) in Visbal et al. (2014). By redshift $\approx 24-26$, many of the models capable of generating the EDGES feature bring the minimum halo mass above the atomic cooling threshold, which would severely curtail Pop-III seed formation, serving as a potential mechanism for shutting down black hole production before $z \approx 16$ and satisfying constraints on black hole production and radiative backgrounds. Pop-III shut down from black-holegenerated LW feedback before $z \approx 20$ stands in contrast to models in which the LW background only comes from Pop-III stars themselves. Scenarios without black holes have been found to allow for star formation in pristine molecular cooling halos until $z \lesssim 10$ (Jaacks et al. 2018; Mebane et al. 2018). The formula from Visbal et al. (2014), which we have used to predict $T_{\text {crit, }}$, ignores the potentially countervailing feedback from X-rays. Since X-rays generate free electrons, they work to catalyze $\mathrm{H}_{2}$ formation and decrease $T_{\text {crit }}$. The actual shutdown redshift for Pop-III formation is therefore somewhat lower than what our simple analysis predicts and we defer a more detailed analysis to future work.

\subsection{Contribution to the Cosmic Infrared Background}

We also check our model's contribution to the cosmic infrared background at $3.6 \mu \mathrm{m}$ using a procedure similar to what is described in Section 3.5. We assume that our black holes have a luminosity at $2500 \AA$ given by Equation (12) with a spectral index of -0.65 redward of $912 \AA$. For this treatment, all of our models predict IR fluxes at $3.6 \mu \mathrm{m}$ (integrated across the IRAC filter width of $\approx 1 \mu \mathrm{m}$ ) that are $\lesssim 10^{-4} \mathrm{nW} \mathrm{m}^{-2} \mathrm{~Hz}^{-1} \mathrm{sr}^{-1}$. This is well below the typical values that are supposedly from cosmic dawn sources, of $\sim 1 \mathrm{nW} \mathrm{m}{ }^{-2} \mathrm{sr}^{-1}$ (Kashlinsky et al. 2007). This calculation ignores reprocessed IR emission caused by the absorption of UV and X-ray photons by any obscuring clouds. We leave the study of reprocessed radiation, which might greatly exceed the unobscured quasar emission considered here, for future investigations.

\section{Discussion}

While we have shown that black holes can produce a CMBlevel $21 \mathrm{~cm}$ background by $z \approx 17$, under optimistic assumptions of accretion rates and present-day correlations between $\mathrm{X}$-ray and radio luminosities, there are still significant issues that a black-hole-driven model must overcome before it is considered a serious explanation for the EDGES excess.

First, our accretion model produces copious amounts of $\mathrm{X}$-rays that would raise the temperature of the IGM (and potentially contribute significantly to reionization). Any such rise in $T_{s}$ would erase the gains made in the amplitude of absorption unless energy deposition of the X-rays was delayed until redshift $z \lesssim 16$, where EDGES observes the rise out of the trough. Fialkov et al. (2014; also see Mesinger et al. (2013)) discussed a heating scenario in which a hard $\mathrm{X}$-ray spectrum due to obscuration at $\lesssim 1 \mathrm{keV}$ could reduce their energy and delay energy deposition, significantly enhancing the amplitude of the absorption feature. Thus, one possibility is that the progenitors to SMBHs were born in heavily obscured environments that prevented the escape of X-ray and UV photons but still allowed for Ly $\alpha$ to strongly couple the gas to 
the adiabatically cooled IGM. Tanaka et al. (2016) came to a similar conclusion, as they noticed that unobscured X-ray emission from Pop-III black holes would erase the absorption feature.

We may estimate the required obscuration to keep the IGM unheated over the duration of the EDGES feature by considering the (physical) mean-free path of an X-ray in the IGM:

$$
\lambda_{\mathrm{X}} \approx 1.56 x_{\mathrm{H} \mathrm{I}}^{-1}\left(\frac{1+z}{17}\right)^{-3}\left(\frac{E}{500 \mathrm{eV}}\right)^{2.6} \mathrm{Mpc} .
$$

If X-rays with energies below $E_{\min }$ are absorbed locally, energy deposition (and heating) of the IGM will be delayed by $\tau_{X}=\lambda_{X}\left(E_{\min }\right) / c$.

Provided that the gas remains mostly unheated over the duration of the absorption trough, and the upturn at $z \approx 14$ is driven by rapid heating, the shape and duration of the absorption trough reported by EDGES actually provides us with constraining information on the local column depths of black holes providing the radio background. The width of the trough is $\approx 100 \mathrm{Myr}$. Solving for $c \times 100 \mathrm{Myr}=\lambda_{\mathrm{X}}\left(E_{\mathrm{min}}\right)$ yields $E_{\text {min }} \approx 1.7 \mathrm{keV}$. The column depth required to obscure X-rays up to $E_{\min } \approx 1.7 \mathrm{keV}$ can be obtained by setting $N_{\mathrm{H}} \sim \sigma_{\mathrm{H}}^{-1}(1.7 \mathrm{keV}) \sim 5 \times 10^{23} \mathrm{~cm}^{-2}$, where $\sigma_{\mathrm{H}}$ is the photoionization cross section of hydrogen. Models explaining the X-ray background from AGNs at lower redshift often invoke similar obscuration column depths (Fabian 1999), requiring that the gas forms a dense, efficiently cooled spheroid around the black hole. At high redshift, the obscuration eventually might be disrupted by the black hole's emission, once it has grown to a sufficiently large size, which might hasten the evolution of $T_{s}$ and explain the very rapid elimination of the absorption feature by $z=14$. We check that such column depths would not obscure radio emission by assuming that the gas with a fixed column depth forms a spherical cloud with constant density around the black hole. Even if the gas were highly ionized, we find that the plasma frequencies for such column depths do not exceed $\approx 10 \mathrm{MHz}$.

Our requirement that the IGM remains unheated for $\approx 100 \mathrm{Myr}$ after black holes start emitting imposes the condition that

$$
f_{\mathrm{esc}}\left(N_{\mathrm{H}} \sim 10^{23}\right) \sim \exp \left[10^{23} \mathrm{~cm}^{-2} \sigma_{\mathrm{H}}(13.6 \mathrm{eV})\right] \approx 0 .
$$

Thus, imposing obscuration requirements based on the timing of the signal guarantees that the AGNs do not contribute to reionization until the obscuring gas is cleared away, allowing them to obey the Planck constraint on $\tau$.

Since our black hole seed masses are in the range in which a black hole could form from the direct collapse of a star (without a supernovae), the radiative feedback might be low enough to allow for high obscuration (and efficient growth) of the black hole. At the same time, without any heating, our most optimistic accretion scenario overpredicts the amplitude of the EDGES feature by a factor $\approx 5$, so there are scenarios in which some heating might be tolerated while still recovering the anomalously large absorption feature reported by Bowman et al. (2018).

Second, the models that are capable of producing the reported EDGES amplitude assemble a large fraction of the comoving black hole mass at $z=0$ (between $1 \%$ and $10 \%$ ) by $z \approx 17$, and would likely overproduce the black hole density if exponential growth were allowed to proceed unregulated.
Feedback mechanisms will have to be invoked to suppress the formation of future Pop-III seeds and regulate the growth of the existing black holes beyond $z \approx 16$. Fortunately, it is expected that Pop-III formation naturally ends once the metallicity of gas reaches $\approx 10^{-6}-10^{-3} Z_{\odot}$ (Bromm et al. 2001; Omukai et al. 2005) and while a number of models show that Pop-III formation can continue down to redshifts of $z \lesssim 10$, we have shown that the additional Lyman-Werner feedback generated by rapidly accreting Pop-III black holes capable of producing the EDGES feature is large enough to shutdown black hole seed formation at $z \sim 20$.

Obscuration of the Pop-III black holes necessary to prevent significant heating and ionization in the IGM would naturally lead to large amounts of energy being deposited within their local environments, providing an additional regulation mechanism. Detailed modeling to determine whether a combination of obscuration and feedback can both delay heating and shutdown accretion in a manner that explains the EDGES absorption feature is left to future work.

Third, while only our Pop-III model appears to produce a sufficiently bright radio background to explain the EDGES feature, detailed simulations have found that heating and photoionization feedback tend to prevent Pop-III remnants from achieving high accretion rates (e.g., Alvarez et al. 2009). Hence, rapid accretion within atomic cooling halos might actually be more plausible than in our Pop-III scenario (for example, models in which $\sim 100 M_{\odot}$ seeds experience supercritical accretion from a thick, obscuring, and radiatively inefficient torus (Volonteri \& Rees 2005)). An argument for atomic cooling halos can also be raised from the timing of the absorption feature, which may start at too low a redshift to be caused by molecular cooling halos (Kaurov et al. 2018). Thus, scenarios where black hole seeds formed in atomic cooling halos in higher abundances than what is predicted in the models that we based our DCBH and cluster scenarios on, are an interesting possibility that should be explored further.

While a radio background might explain the EDGES feature, mechanisms different from those explored here have been suggested for producing this background. They include cosmic rays accelerated in supernovae (Mirocha \& Furlanetto 2018) and instabilities in mini-clusters of axionic dark matter (Fraser et al. 2018). A black-hole-driven scenario might be distinguished from a scenario involving star-forming Galaxies in several ways.

First, Eddington accretion can lead to exponential time evolution of radiative emissivities, significantly faster than scenarios driven by star-forming galaxies, where emissivities evolve proportional to the star formation rate. Second, X-ray emission driven from inverse bremsstrahlung of the same cosmic rays producing the radio emission is known to be significantly softer than X-ray emission from black hole accretion, which would lead to rapid heating and highercontrast spatial fluctuations (Pacucci et al. 2014).

Spatial H I intensity mapping and deep point-source surveys might also be used to distinguish black hole radio emission from a background generated by collapsing axionic miniclusters. In the axion model, the radio background could be generated by halos significantly below the atomic cooling threshold. Since collapsing axionic halos would not generate $\mathrm{X}$-ray emission, the timing and spatial variation between hot/ cold patches of $\mathrm{HI}$ and the radio background would be decoupled and might help to confirm or reject such a model. In 


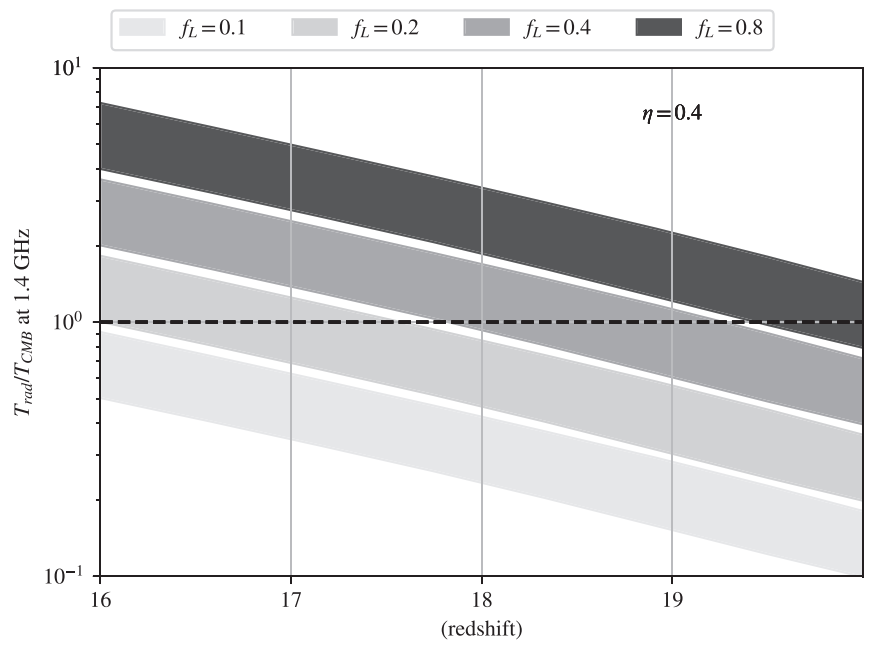

Figure 8. Same as Figure 1 but now only considering Pop-III black holes and fixing $\eta=0.4$, which is an upper limit for thin-disk accretion scenarios with black holes spinning close to their theoretical maximum. Filled regions correspond to $\mathrm{T}_{\mathrm{rad}} / \mathrm{T}_{\mathrm{CMB}}$ between $f_{\text {edd }}=0.1$ and $f_{\text {edd }}=1$ with fixed $f_{L}$. Doubling the radio-loud fraction from what is observed at the present day (i.e., using $f_{L}=0.2$ ) gives us enough emission to produce the EDGES absorption feature.

addition, radio emission from axionic decays might be distinguished by the fact that such emission is intrinsically narrow band.

In our study, we restricted ourselves to scenarios with very rapid accretion driven by low radiative efficiencies $(\eta<0.1)$. Within the thin disk paradigm (Shakura \& Sunyaev 2009), this corresponds to a black hole spin of $a / M_{\mathrm{bh}} \lesssim 0.7$. It is currently unknown what the distribution of initial spins for supermassive black hole progenitors might be and in what fashion they were modified by accretion. Spin evolution through accretion depends heavily on whether accreting material follows prograde or retro-grade orbits and whether accretion episodes are coherent (King et al. 2008). Higher radiative efficiencies than what we examined might still explain the EDGES feature but would require larger radio luminosities per black hole or larger radio-loud fractions than we examined. In Figure 8 we show $T_{\text {rad }} / T_{\mathrm{CMB}}$ for $\eta=0.4$, close to its theoretical maximum, for different values of $f_{L}$ and $f_{\text {edd. }}$. In this radiatively efficient scenario, $f_{L}=0.1$ cannot produce a background large enough for the EDGES feature, but simply doubling the radio-loud fraction (or equivalently the expectation value of radio luminosity) to $f_{L}=0.2$ can.

\section{Conclusion}

In this paper, we have investigated the plausibility that the absorption feature recently detected by the EDGES experiment might have been produced by an additional radio background arising from accretion onto growing black holes during the cosmic dawn. To do this, we combined low-redshift empirical relationships between AGN X-ray luminosities and radio emission, with a semi-analytic framework that creates new seed black holes based on the halo mass function and grows them exponentially through Eddington-limited accretion. With this framework, we have explored plausible radio backgrounds over a broad range of physically motivated seed populations and growth rates. Our main conclusions from this study are as follows:
1. Black holes forming and growing at physically plausible rates can produce a radio background sufficient to explain the amplitude of the EDGES absorption feature while satisfying existing constraints on the soft X-ray background and faint radio source counts.

2. By demanding that the IGM remain unheated over the duration of the EDGES trough $(\sim 100 \mathrm{Myr})$, we find these black holes would need to be obscured with column depths of $N_{\mathrm{H}} \approx 5 \times 10^{23} \mathrm{~cm}^{-2}$. Such large column depths would also prevent these black holes from ionizing the IGM too early.

3. Of the models we considered, none that are limited by the atomic cooling threshold reached more than $10 \%$ of the radio emission needed to explain the EDGES feature. Increasing the DCBH fraction by $\sim 100$ (through enhancements such as baryon-dark matter velocity offsets) could explain the EDGES feature but would come into conflict with the faint source counts constraint in Figure 2, though a steeper spectral index or reducing $f_{\text {seed }}$ in exchange for a further increase in $f_{\text {halo }}$ can mitigate this issue. The cluster-collapse scenario we considered is still viable if a $\sim 10 \times$ larger fraction $(\approx 50 \%)$ of halos hosted such seeds. It is also possible that a black hole population arising from a combination of all three mechanisms in a wide range of halo masses could produce a sufficiently bright radio background.

4. In order to avoid overproducing the local black hole population and radiative backgrounds at low redshift, the emission and growth of these black holes would need to be curtailed at $z \lesssim 16$. We have shown that the LW background generated by the black holes themselves is sufficient to shutdown star formation in molecular cooling halos at $z \lesssim 20$, potentially providing the necessary feedback mechanism.

While we have diligently checked the implications of our model against observational constraints of radio counts, the CXB, CIB, and CMB optical depth, we caution our readers to heed the caveats in Section 4.

The authors thank Judd Bowman, Adam Lidz, Rennan Barkana, Anastasia Fialkov, and Jordan Mirocha for useful discussions, along with Florian Bolgar for correcting several numerical errors. Calculations in this work were performed using the Colossus library (Diemer 2017). A.E.W.'s contribution was supported by an appointment to the NASA Postdoctoral Program at the California Institute of Technology Jet Propulsion Laboratory. Part of the research was carried out at the Jet Propulsion Laboratory, California Institute of Technology, under a contract with the National Aeronautics and Space Administration. R.A.M. was supported by the NASA Solar System Exploration Virtual Institute cooperative agreement 80ARC017M0006.

\section{ORCID iDs}

A. Ewall-Wice (1) https://orcid.org/0000-0002-0086-7363

R. A. Monsalve (i) https://orcid.org/0000-0002-3287-2327

\section{References}

Abel, T., Anninos, P., Zhang, Y., \& Norman, M. L. 1997, NewA, 2, 181 Abel, T., Bryan, G. L., \& Norman, M. L. 2002, Sci, 295, 93

Agarwal, B., Khochfar, S., Johnson, J. L., et al. 2012, MNRAS, 425, 2854 
Alexander, T., \& Natarajan, P. 2014, Sci, 345, 1330

Ali, Z. S., Parsons, A. R., Zheng, H., et al. 2015, ApJ, 809, 61

Alvarez, M. A., Wise, J. H., \& Abel, T. 2009, ApJL, 701, L133

Bañados, E., Venemans, B. P., Mazzucchelli, C., et al. 2018, Natur, 553, 473

Bañados, E., Venemans, B. P., Morganson, E., et al. 2015, ApJ, 804, 118

Barkana, R. 2018, Natur, 555, 71

Barkana, R., \& Loeb, A. 2001, PhR, 349, 125

Beardsley, A. P., Hazelton, B. J., Sullivan, I. S., et al. 2016, ApJ, 833, 102

Begelman, M. C., Volonteri, M., \& Rees, M. J. 2006, MNRAS, 370, 289

Bernardi, G., Zwart, J. T. L., Price, D., et al. 2016, MNRAS, 461, 2847

Biermann, P. L., Nath, B. B., Caramete, L. I., et al. 2014, MNRAS, 441, 1147

Bolgar, F., Eames, E., Hottier, C., \& Semelin, B. 2018, MNRAS, 478, 5564

Bowman, J. D., \& Rogers, A. E. E. 2010, Natur, 468, 796

Bowman, J. D., Rogers, A. E. E., Monsalve, R. A., Mozdzen, T. J., \& Mahesh, N. 2018, Natur, 555, 67

Bridge, C. R., Teplitz, H. I., Siana, B., et al. 2010, ApJ, 720, 465

Bromm, V., Coppi, P. S., \& Larson, R. B. 2002, ApJ, 564, 23

Bromm, V., Ferrara, A., Coppi, P. S., \& Larson, R. B. 2001, MNRAS, 328,969

Bromm, V., \& Loeb, A. 2003, ApJ, 596, 34

Cappelluti, N., Ranalli, P., Roncarelli, M., et al. 2012, MNRAS, 427, 651

Carilli, C. L., Gnedin, N. Y., \& Owen, F. 2002, ApJ, 577, 22

Choudhury, T. R., \& Ferrara, A. 2006, MNRAS, 371, L55

Ciardi, B., Labropoulos, P., Maselli, A., et al. 2013, MNRAS, 428, 1755

Cirasuolo, M., Celotti, A., Magliocchetti, M., \& Danese, L. 2003, MNRAS, 346, 447

Clark, P. C., Glover, S. C. O., Smith, R. J., et al. 2011, Sci, 331, 1040

Cohen, A., Fialkov, A., Barkana, R., \& Lotem, M. 2017, MNRAS, 472, 1915

Condon, J. J., Cotton, W. D., Fomalont, E. B., et al. 2012, ApJ, 758, 23

Das, A., Mesinger, A., Pallottini, A., Ferrara, A., \& Wise, J. H. 2017, MNRAS, 469, 1166

Davies, M. B., Miller, M. C., \& Bellovary, J. M. 2011, ApJL, 740, L42

DeBoer, D. R., Parsons, A. R., Aguirre, J. E., et al. 2017, PASP, 129, 045001

Devecchi, B., \& Volonteri, M. 2009, ApJ, 694, 302

Diemer, B. 2017, arXiv:1712.04512

Dijkstra, M., Ferrara, A., \& Mesinger, A. 2014, MNRAS, 442, 2036

Dillon, J. S., Liu, A., Williams, C. L., et al. 2014, PhRvD, 89, 023002

Donoso, E., Best, P. N., \& Kauffmann, G. 2009, MNRAS, 392, 617

Dunn, G., Bellovary, J., Holley-Bockelmann, K., Christensen, C., \& Quinn, T. 2018, ApJ, 861, 39

Ewall-Wice, A., Dillon, J. S., Hewitt, J. N., et al. 2016, MNRAS, 460, 4320

Ewall-Wice, A., Dillon, J. S., Mesinger, A., \& Hewitt, J. 2014, MNRAS, 441, 2476

Fabian, A. C. 1999, MNRAS, 308, L39

Fakhouri, O., Ma, C.-P., \& Boylan-Kolchin, M. 2010, MNRAS, 406, 2267

Falcke, H., Körding, E., \& Markoff, S. 2004, A\&A, 414, 895

Fan, X., Strauss, M. A., Schneider, D. P., et al. 2003, AJ, 125, 1649

Feng, C., \& Holder, G. 2018, ApJL, 858, L17

Ferrara, A., Salvadori, S., Yue, B., \& Schleicher, D. 2014, MNRAS, 443, 2410

Fialkov, A., Barkana, R., \& Cohen, A. 2018, PhRvL, 121, 011101

Fialkov, A., Barkana, R., Tseliakhovich, D., \& Hirata, C. M. 2012, MNRAS, 424, 1335

Fialkov, A., Barkana, R., \& Visbal, E. 2014, Natur, 506, 197

Fialkov, A., Cohen, A., Barkana, R., \& Silk, J. 2017, MNRAS, 464, 3498

Fixsen, D. J., Kogut, A., Levin, S., et al. 2011, ApJ, 734, 5

Fraser, S., Hektor, A., Hütsi, G., et al. 2018, PhLB, 785, 159

Furlanetto, S. R. 2006, MNRAS, 370, 1867

Furlanetto, S. R., \& Loeb, A. 2002, ApJ, 579, 1

Furlanetto, S. R., Oh, S. P., \& Briggs, F. H. 2006, PhR, 433, 181

Ghisellini, G., Celotti, A., Tavecchio, F., Haardt, F., \& Sbarrato, T. 2014, MNRAS, 438, 2694

Ghisellini, G., Haardt, F., Ciardi, B., et al. 2015, MNRAS, 452, 3457

Grazian, A., Giallongo, E., Paris, D., et al. 2017, A\&A, 602, A18

Greif, T. H., Bromm, V., Clark, P. C., et al. 2012, MNRAS, 424, 399

Greif, T. H., Springel, V., White, S. D. M., et al. 2011a, ApJ, 737, 75

Greif, T. H., Springel, V., White, S. D. M., et al. 2011b, ApJ, 737, 75

Greif, T. H., White, S. D. M., Klessen, R. S., \& Springel, V. 2011c, ApJ, 736,147

Haiman, Z., \& Bryan, G. L. 2006, ApJ, 650, 7

Haiman, Z., \& Loeb, A. 2001, ApJ, 552, 459

Haiman, Z., Quataert, E., \& Bower, G. C. 2004, ApJ, 612, 698

Haiman, Z., Rees, M. J., \& Loeb, A. 1997, ApJ, 476, 458

Haiman, Z., Thoul, A. A., \& Loeb, A. 1996, ApJ, 464, 523

Heinrich, C., \& Hu, W. 2018, PhRvD, 98, 063514

Hickox, R. C., \& Markevitch, M. 2006, ApJ, 645, 95

Hirano, S., Hosokawa, T., Yoshida, N., \& Kuiper, R. 2017, Sci, 357, 1375
Hirano, S., Hosokawa, T., Yoshida, N., Omukai, K., \& Yorke, H. W. 2015, MNRAS, 448, 568

Hosokawa, T., Hirano, S., Kuiper, R., et al. 2016, ApJ, 824, 119

Ivezić, Ž, Menou, K., Knapp, G. R., et al. 2002, AJ, 124, 2364

Ivezić, Z., Richards, G., Hall, P., et al. 2004, in ASP Conf. Ser. 311, AGN

Physics with the Sloan Digital Sky Survey, ed. G. T. Richards \& P. B. Hall (San Francisco, CA: ASP), 347

Izotov, Y. I., Schaerer, D., Thuan, T. X., et al. 2016, MNRAS, 461, 3683

Jaacks, J., Thompson, R., Finkelstein, S. L., \& Bromm, V. 2018, MNRAS, 475, 4396

Jacobs, D. C., Hazelton, B. J., Trott, C. M., et al. 2016, ApJ, 825, 114

Jacobs, D. C., Pober, J. C., Parsons, A. R., et al. 2015, ApJ, 801, 51

Jiang, L., Fan, X., Ivezić, Ž, et al. 2007, ApJ, 656, 680

Johnson, J. L., Greif, T. H., \& Bromm, V. 2008, MNRAS, 388, 26

Johnson, J. L., Whalen, D. J., Fryer, C. L., \& Li, H. 2012, ApJ, 750, 66

Johnson, J. L., Whalen, D. J., Li, H., \& Holz, D. E. 2013, ApJ, 771, 116

Kashlinsky, A., Arendt, R. G., Mather, J., \& Moseley, S. H. 2007, ApJL, 654, L1

Kaurov, A. A., Venumadhav, T., Dai, L., \& Zaldarriaga, M. 2018, ApJL, 864, L15

Kellerman, K. I., Sramek, R., Schmidt, M., Shaffer, D. B., \& Green, R. 1989, AJ, 98, 1195

King, A. R., Pringle, J. E., \& Hofmann, J. A. 2008, MNRAS, 385, 1621

Kohn, S., Chichura, P. M., Igarashi, A. S., et al. 2018, ApJ, submitted (arXiv: 1802.04151)

Lehmer, B. D., Xue, Y. Q., Brandt, W. N., et al. 2012, ApJ, 752, 46

Lodato, G., \& Natarajan, P. 2006, MNRAS, 371, 1813

Lusso, E., Comastri, A., Vignali, C., et al. 2010, A\&A, 512, A34

Lusso, E., Worseck, G., Hennawi, J. F., et al. 2015, MNRAS, 449, 4204

Ma, X., Kasen, D., Hopkins, P. F., et al. 2015, MNRAS, 453, 960

Mack, K. J., \& Wyithe, J. S. B. 2012, MNRAS, 425, 2988

Madau, P., \& Haardt, F. 2015, ApJL, 813, L8

Madau, P., Haardt, F., \& Dotti, M. 2014, ApJL, 784, L38

Madau, P., Meiksin, A., \& Rees, M. J. 1997, ApJ, 475, 429

Madau, P., \& Rees, M. J. 2001, ApJL, 551, L27

Madau, P., \& Shull, J. M. 1996, ApJ, 457, 551

McCammon, D., Almy, R., Apodaca, E., et al. 2002, ApJ, 576, 188

McQuinn, M. 2016, ARA\&A, 54, 313

Mebane, R. H., Mirocha, J., \& Furlanetto, S. R. 2018, MNRAS, 479, 4544

Merloni, A., Heinz, S., \& di Matteo, T. 2003, MNRAS, 345, 1057

Merritt, D., \& Ferrarese, L. 2001, MNRAS, 320, L30

Mesinger, A., Ferrara, A., \& Spiegel, D. S. 2013, MNRAS, 431, 621

Micheva, G., Iwata, I., \& Inoue, A. K. 2017, MNRAS, 465, 302

Millea, M., \& Bouchet, F. 2018, A\&A, 617, A96

Milosavljević, M., Bromm, V., Couch, S. M., \& Oh, S. P. 2009, ApJ, 698, 766

Mirocha, J., \& Furlanetto, S. R. 2018, MNRAS, submitted (arXiv:1803.03272)

Monsalve, R. A., Rogers, A. E. E., Bowman, J. D., \& Mozdzen, T. J. 2017, ApJ, 847, 64

Morales, M. F., \& Wyithe, J. S. B. 2010, ARA\&A, 48, 127

Mortlock, D. J., Warren, S. J., Venemans, B. P., et al. 2011, Natur, 474, 616

Muñoz, J. B., Kovetz, E. D., \& Ali-Haïmoud, Y. 2015, PhRvD, 92, 083528

Muñoz, J. B., \& Loeb, A. 2018, Natur, 557, 684

Nandra, K., \& Pounds, K. A. 1994, MNRAS, 268, 405

Omukai, K., Tsuribe, T., Schneider, R., \& Ferrara, A. 2005, ApJ, 626, 627

Paciga, G., Albert, J. G., Bandura, K., et al. 2013, MNRAS, 433, 639

Pacucci, F., Ferrara, A., Volonteri, M., \& Dubus, G. 2015, MNRAS, 454, 3771

Pacucci, F., Mesinger, A., Mineo, S., \& Ferrara, A. 2014, MNRAS, 443, 678

Page, K. L., Reeves, J. N., O'Brien, P. T., \& Turner, M. J. L. 2005, MNRAS, 364, 195

Parsons, A. R., Liu, A., Aguirre, J. E., et al. 2014, ApJ, 788, 106

Patil, A. H., Yatawatta, S., Koopmans, L. V. E., et al. 2017, ApJ, 838, 65

Peebles, P. J. E. 1993, Principles of Physical Cosmology (Princeton, NJ: Princeton Univ. Press)

Piconcelli, E., Jimenez-Bailón, E., Guainazzi, M., et al. 2005, A\&A, 432, 15

Planck Collaboration, Ade, P. A. R., Aghanim, N., et al. 2016, A\&A, 594, A13

Pritchard, J. R., \& Loeb, A. 2012, RPPh, 75, 086901

Reeves, J. N., \& Turner, M. J. L. 2000, MNRAS, 316, 234

Rhook, K. J., \& Haehnelt, M. G. 2006, MNRAS, 373, 623

Saxena, A., Röttgering, H. J. A., \& Rigby, E. E. 2017, MNRAS, 469, 4083

Semelin, B. 2016, MNRAS, 455, 962

Shakura, N. I., \& Sunyaev, R. A. 2009, A\&A, 500, 33

Shang, C., Bryan, G. L., \& Haiman, Z. 2010, MNRAS, 402, 1249

Shankar, F., Crocce, M., Miralda-Escudé, J., Fosalba, P., \& Weinberg, D. H. 2010, ApJ, 718, 231

Shankar, F., Weinberg, D. H., \& Miralda-Escudé, J. 2009, ApJ, 690, 20

Sharma, P. 2018, MNRAS, 481, L6 
Shen, Y., \& Kelly, B. C. 2012, ApJ, 746, 169

Singal, J., Haider, J., Ajello, M., et al. 2018a, PASP, 130, 036001

Singal, J., Haider, J., Ajello, M., et al. 2018b, PASP, 130, 036001

Singh, S., Subrahmanyan, R., Udaya Shankar, N., et al. 2017, ApJ, 858, 54

Sobacchi, E., \& Mesinger, A. 2014, MNRAS, 440, 1662

Sokolowski, M., Wayth, R. B., Tremblay, S. E., et al. 2015, ApJ, 813, 18

Stacy, A., Bromm, V., \& Loeb, A. 2011, ApJ, 730, L1

Stacy, A., Greif, T. H., \& Bromm, V. 2010, MNRAS, 403, 45

Stone, N. C., Küpper, A. H. W., \& Ostriker, J. P. 2017, MNRAS, 467, 4180

Tanaka, T. L., O'Leary, R. M., \& Perna, R. 2016, MNRAS, 455, 2619

Tashiro, H., Kadota, K., \& Silk, J. 2014, PhRvD, 90, 083522

Tegmark, M., Silk, J., Rees, M. J., et al. 1997, ApJ, 474, 1

Tinker, J., Kravtsov, A. V., Klypin, A., et al. 2008, ApJ, 688, 709

Trott, C. M., Pindor, B., Procopio, P., et al. 2016, ApJ, 818, 139

Vanzella, E., de Barros, S., Vasei, K., et al. 2016, ApJ, 825, 41
Vasei, K., Siana, B., Shapley, A. E., et al. 2016, ApJ, 831, 38

Vernstrom, T., Scott, D., Wall, J. V., et al. 2014, MNRAS, 440, 2791

Visbal, E., Haiman, Z., \& Bryan, G. L. 2015, MNRAS, 453, 4456

Visbal, E., Haiman, Z., Terrazas, B., Bryan, G. L., \& Barkana, R. 2014, MNRAS, 445, 107

Volonteri, M., \& Rees, M. J. 2005, ApJ, 633, 624

Volonteri, M., \& Rees, M. J. 2006, ApJ, 650, 669

Volonteri, M., Silk, J., \& Dubus, G. 2015, ApJ, 804, 148

Voytek, T. C., Natarajan, A., Jáuregui García, J. M., Peterson, J. B., \& López-Cruz, O. 2014, ApJL, 782, L9

Wang, R., Wu, X.-B., \& Kong, M.-Z. 2006, ApJ, 645, 890

Wilman, R. J., Miller, L., Jarvis, M. J., et al. 2008, MNRAS, 388, 1335

Wu, X.-B., Wang, F., Fan, X., et al. 2015, Natur, 518, 512

Yoon, S. C., Dierks, A., \& Langer, N. 2012, A\&A, 542, A113

Yoshida, N., Omukai, K., \& Hernquist, L. 2008, Sci, 321, 669 